\title{
Enforcing the linear behavior of the total energy with hybrid functionals: Implications for charge transfer, interaction energies, and the random-phase approximation
}

\author{
Viktor Atalla, ${ }^{1, *}$ Igor Ying Zhang, ${ }^{1}$ Oliver T. Hofmann, ${ }^{1,2}$ Xinguo Ren, ${ }^{1,3}$ Patrick Rinke, ${ }^{1,4}$ and Matthias Scheffler ${ }^{1}$ \\ ${ }^{1}$ Fritz-Haber-Institut der Max-Planck-Gesellschaft, Faradayweg 4-6, D-14195 Berlin, Germany \\ ${ }^{2}$ Institut fur Festkörperphysik, Technische Universität Graz, Petersgasse 16, 8010 Graz, Austria \\ ${ }^{3}$ Key Laboratory of Quantum Information, University of Science and Technology of China, Hefei, 230026, Anhui, Peoples Republic of China \\ ${ }^{4}$ COMP/Department of Applied Physics, Aalto University, P.O. Box 11100, FI-00076 Aalto, Finland
}

(Received 6 December 2015; revised manuscript received 6 May 2016; published 19 July 2016)

\begin{abstract}
We obtain the exchange parameter of hybrid functionals by imposing the fundamental condition of a piecewise linear total energy with respect to electron number. For the Perdew-Burke-Ernzerhof (PBE) hybrid family of exchange-correlation functionals (i.e., for an approximate generalized Kohn-Sham theory) this implies that (i) the highest occupied molecular orbital corresponds to the ionization potential $(I)$, (ii) the energy of the lowest unoccupied molecular orbital corresponds to the electron affinity $(A)$, and (iii) the energies of the frontier orbitals are constant as a function of their occupation. In agreement with a previous study [N. Sai et al., Phys. Rev. Lett. 106, 226403 (2011)], we find that these conditions are met for high values of the exact exchange admixture $\alpha$ and illustrate their importance for the tetrathiafulvalene-tetracyanoquinodimethane complex for which standard density functional theory functionals predict artificial electron transfer. We further assess the performance for atomization energies and weak interaction energies. We find that atomization energies are significantly underestimated compared to PBE or PBE0, whereas the description of weak interaction energies improves significantly if a $1 / R^{6}$ van der Waals correction scheme is employed.
\end{abstract}

DOI: 10.1103/PhysRevB.94.035140

\section{INTRODUCTION}

Hybrid exchange-correlation (XC) functionals have significantly improved the description of molecules and solids [1-5]. They incorporate a fraction $\alpha$ of exact-exchange energy either in a global or in a range separated fashion. One common global hybrid functional is the Perdew-Burke-Ernzerhof (PBE) hybrid functional [6,7], which approximates the XC energy by

$$
E_{\mathrm{XC}}(\alpha)=\alpha E_{\mathrm{X}}^{\mathrm{EX}}+(1-\alpha) E_{\mathrm{X}}^{\mathrm{PBE}}+E_{\mathrm{C}}^{\mathrm{PBE}} .
$$

In this parametrization, a fraction $\alpha$ of exact exchange $\left(E_{\mathrm{X}}^{\mathrm{EX}}\right)$ is combined with the complement $1-\alpha$ of PBE exchange $\left(E_{\mathrm{X}}^{\mathrm{PBE}}\right)$. The correlation energy $\left(E_{\mathrm{C}}^{\mathrm{PBE}}\right)$ is approximated on the PBE level and is independent of $\alpha$. There are several strategies to determine $\alpha$ : a performance driven avenue by fitting to thermochemical data sets [8,9], theoretical models of the adiabatic connection path $[7,10]$, or by requiring that the ensuing functional obeys certain properties that are known for the exact XC functional.

One common practice is to require the HOMO-I condition, which states that the eigenvalue of the highest molecular orbital (HOMO) corresponds to the ionization potential ( $I$ ). $\alpha$ could then be obtained by requiring that the HOMO level matches the $I$ obtained by the total energy difference of the neutral and the positively charged molecule ( $\triangle \mathrm{SCF}$ approach). Alternatively, the HOMO- $I$ condition can be incorporated by means of the single-particle Green's function. In this approach, $\alpha$ is determined by requiring that the self-energy correction to the HOMO level within the $G_{0} W_{0}$ approximation [11] is minimized. Eventually, this also fixes the starting point dependence of the perturbative $G W$ approximation [12-14].

\footnotetext{
*Present address: Qudosoft, Schwedterstrasse 263, D-10119 Berlin, Germany.
}

Specifically, the optimum fraction of exact exchange $\alpha^{*}$ is obtained by minimizing the self-energy correction to the HOMO level $[12,15]$

$$
\alpha^{*}=\underset{\alpha}{\arg \min }\left|\left\langle\psi_{\mathrm{H}}(\alpha)\left|\Sigma(\alpha)-V_{\mathrm{xc}}^{\mathrm{DFT}}(\alpha)\right| \psi_{\mathrm{H}}(\alpha)\right\rangle\right|,
$$

where $\psi_{\mathrm{H}}(\alpha)$ is the HOMO, $\Sigma$ is the self-energy in the $G_{0} W_{0}$ approximation, and $\Sigma^{\mathrm{DFT}}$ are the exchange and correlation potentials within the generalized Kohn-Sham (GKS) framework [16]. For the PBE hybrid functional, this takes the form

$$
V_{\mathrm{XC}}^{\mathrm{DFT}}(\alpha)=\alpha v_{x}^{\mathrm{NL}}+(1-\alpha) v_{x}^{\mathrm{PBE}}+v_{c}^{\mathrm{PBE}},
$$

where $v_{x}^{\mathrm{NL}}$ is the nonlocal exact exchange potential, $v_{c}^{\mathrm{PBE}}$ the local correlation, and $v_{x}^{\mathrm{PBE}}$ the local exchange potential in the PBE approximation [6]. The corresponding hybrid functional will then be called $\operatorname{PBEh}\left(\alpha^{*}\right)$, i.e., a PBE hybrid functional that employs $\alpha^{*}$ of exact exchange according to Eqs. (1) and (2).

In this work, we investigate the relationship between this choice of $\alpha^{*}$ (i.e., via the $G_{0} W_{0}$ self-energy) and the $\triangle \mathrm{SCF}$ approach. We also discuss other possible choices of $\alpha$ such that certain properties of the exact functional are obeyed, most notably a theorem by Perdew and coauthors, which states that the total energy is piecewise linear between integer charges [17]. We call approximate functionals that violate this condition to suffer from the deviation of straight line error (DSLE) and approximate functionals that are piecewise linear DSLE free. A remarkable consequence of the linearity condition is that the eigenvalue of the partially filled frontier orbitals must be constant, which is true for the exact functional and for DSLE-free approximate functionals. $\alpha$ could thus be obtained by the requirement that the eigenvalues of the partially filled frontier orbitals are independent of their occupation. Furthermore, for DSLE-free approximate functionals that are explicit differentiable functionals of the density matrix it has been recently shown that the eigenvalue of the lowest 
unoccupied molecular orbital (LUMO) corresponds to the electron affinity $(A)$ [18]. This implies an alternative criterion for the choice of $\alpha$, i.e., the LUMO- $A$ condition. The HOMO-I and the LUMO- $A$ conditions were applied in previous work to restore the linearity condition [19-23]. This route has been predominantly followed with the long-range separated Baer-Neuhauser-Livshiz (BNL) hybrid functional [24,25].

In this paper, we illustrate these concepts for the tetrathiafulvalene (TTF) and tetracyanoquinodimethane (TCNQ) molecules. We show that the choice of $\alpha^{*}$ according to Eq. (2) gives very similar, albeit slightly larger, values than criterions that are based on the piecewise linearity condition. We demonstrate that the DSLE in standard functionals induces artificial electron transfer from TTF to TCNQ in the noninteracting limit, which may be remedied by $\operatorname{PBEh}\left(\alpha^{*}\right)$.

While $\operatorname{PBEh}\left(\alpha^{*}\right)$ HOMO levels are in good agreement with experiment and reference data [12], we further assess its performance for weak interaction energies (S66 test set by Hobza and co-workers [26]), atomization energies (G2-1 test set by Curtiss et al. [27]), and chemical reaction barrier heights (HTBH and NHTBH test set by Truhlar and coworkers $[28,29])$. For weak van der Waals (vdW) interactions, we examine the compatibility of $\operatorname{PBEh}\left(\alpha^{*}\right)$ with the vdW correction scheme by Tkatchenko and Scheffler (TS) [30]. Alternatively, vdW interactions are automatically included in advanced density functionals based on the random-phase approximation (RPA). Most notable is the recent development of renormalized second-order perturbation theory (rPT2) [31,32]. It adds second-order screened exact exchange (SOSEX) and renormalized single excitation (rSE) from second-order Rayleigh-Schrödinger perturbation theory to the RPA correlation energy. RPA and rPT2 are evaluated perturbatively based on density functional theory (DFT) input orbitals. We investigate the compatibility of rPT2 with PBE, $\operatorname{PBE0}$, and $\operatorname{PBEh}\left(\alpha^{*}\right)$ by means of a comparative assessment.

This paper is organized as follows. In Sec. II, we give a comprehensive review of known properties of the exact and approximate functional that are of importance for charge transfer systems. In Sec. III, we review existing methods that rectify deficiencies of standard approximations to the $\mathrm{XC}$ functionals. In Sec. IV, we introduce our computational approach. In Sec. V, we investigate TTF, TCNQ, and the TTF-TCNQ complex, and in Sec. VI, we present benchmark data for weak interaction energies, atomization energies, and barrier heights. An error analysis is performed in Sec. VII.

\section{EXACT AND APPROXIMATE PROPERTIES OF THE XC FUNCTIONAL}

In this section we review properties of the exact XC functional and their ramifications for approximate functionals. The informed reader is encouraged to proceed directly to Sec. IV, in which we introduce our computational approach or to Sec. V, in which we illustrate and analyze the DSLE and its consequences for the prototypical TTF-TCNQ donor acceptor complex.

Linearity condition. In a seminal paper, Perdew et al. discuss a fundamental property of the exact XC functional, which states that the total energy $(E)$ is continuous and piecewise linear between integer charges in the grand canon-

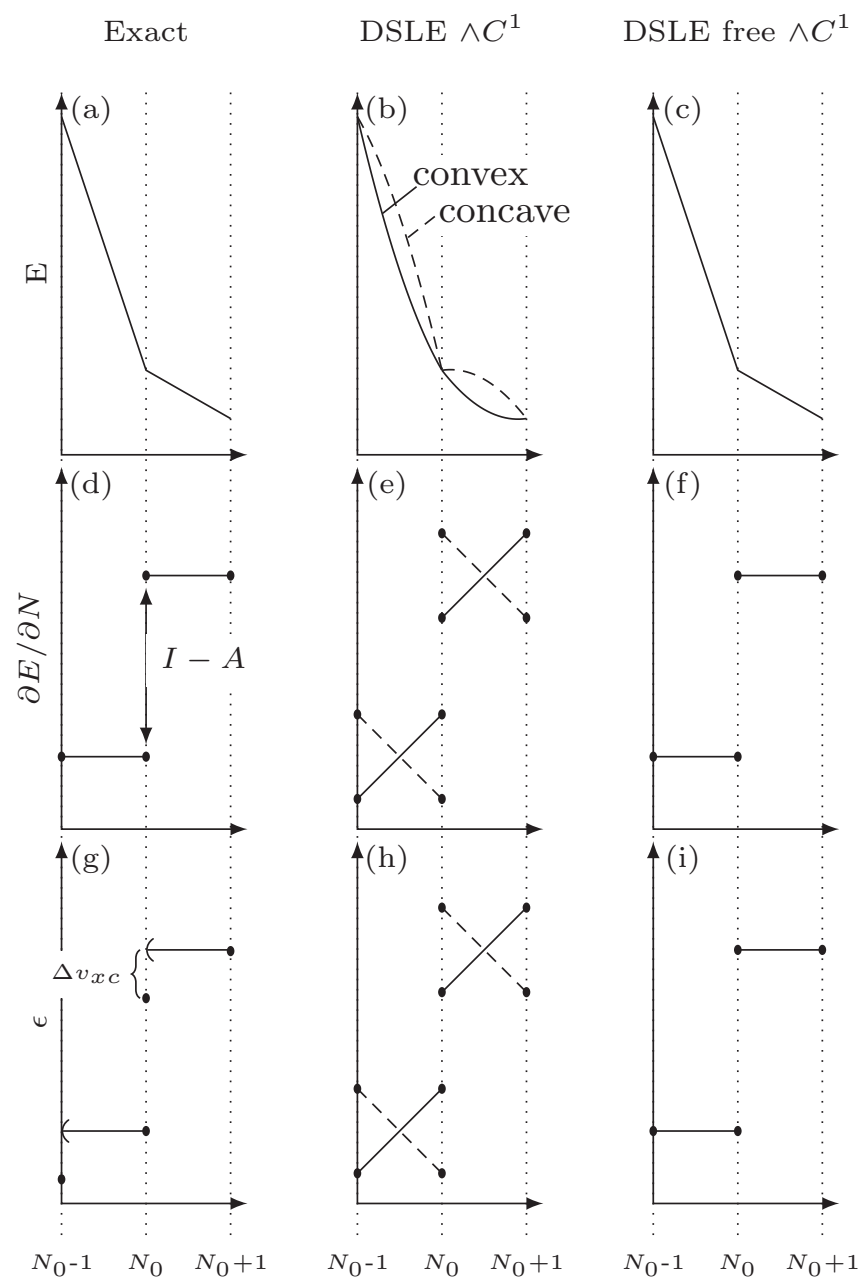

FIG. 1. Summary of properties of the exact functional (left row), approximate functionals that suffer from the DSLE (middle row), and approximate functionals that are DSLE free (right row). The top panel shows the total energy, the middle panel the derivative of the total energy with respect to particle number, and the bottom panel the corresponding eigenvalues. Note that for mathematical clarity, there are two functions in each graph in the last two panels. They correspond to the two closed domains of definition $\left[N_{0}-1, N_{0}\right]$ and $\left[N, N_{0}+1\right]$, whereas the boundary values correspond to the one-sided limits. For example, $\partial E / \partial N\left(N_{0}\right)=\partial E / \partial N^{-}\left(N_{0}\right)$ if $E$ is considered over $\left[N_{0}-\right.$ $\left.1, N_{0}\right]$ and $\partial E / \partial N^{+}\left(N_{0}\right)$ if $E$ is considered over $\left[N_{0}, N_{0}+1\right] . C^{1}$ refers to approximate functionals that are explicit and differentiable functionals of the first-order density matrix.

ical ensemble [17]. For fractional charge $N=N_{0}+\omega$ with $0 \leqslant \omega \leqslant 1$ and $N_{0} \in \mathbb{N}$,

$$
E(N)=(1-\omega) E\left(N_{0}\right)+\omega E\left(N_{0}+1\right), \quad \omega \in[0,1]
$$

as illustrated in Fig. 1.

A consequence of piecewise linearity is that the derivative $\partial E / \partial N$, as illustrated in Fig. 2(b), is constant on integer open intervals. From Perdew's theorem the slopes can be readily extracted [Fig. 2(a)]:

$$
\frac{\partial E}{\partial N}=\left\{\begin{array}{lll}
E\left(N_{0}\right)-E\left(N_{0}-1\right) & \text { if } & N \in\left(N_{0}-1, N_{0}\right), \\
E\left(N_{0}+1\right)-E\left(N_{0}\right) & \text { if } & N \in\left(N_{0}, N_{0}+1\right) .
\end{array}\right.
$$




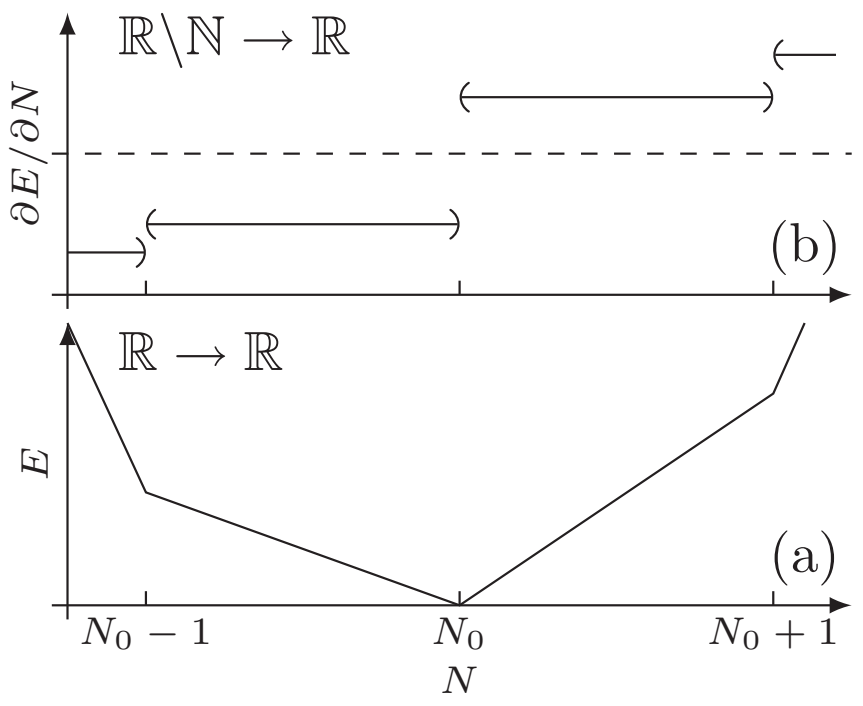

FIG. 2. Illustration of the straight line behavior of the total energy (E) (a) and its derivative (b).

This is just the definition of the ionization potential $(I)$ and the electron affinity ( $A$ ) of the $N_{0}$ electron system. Thus, Eq. (5) may be abbreviated as

$$
\mu(N):=\frac{\partial E}{\partial N}=\left\{\begin{array}{lll}
-I\left(N_{0}\right) & \text { if } & N \in\left(N_{0}-1, N_{0}\right), \\
-A\left(N_{0}\right) & \text { if } \quad N \in\left(N_{0}, N_{0}+1\right),
\end{array}\right.
$$

where on the left side the definition of the chemical potential $\mu$ and the symbols $I$ and $A$ for the ionization potential and the electron affinity were introduced. This relation assumes a fixed external potential, which implies that $I$ and $A$ are the vertical ionization potential and electron affinity (i.e., it does not include geometry relaxation in the excitation process).

Janak's theorem. In Kohn-Sham (KS) DFT, the grand canonical ensemble is mapped onto a noninteracting system with frontier orbitals that may be fractionally occupied. A direct result of Janak's theorem [33,34] and Eq. (5) is that the eigenvalues of the Kohn-Sham frontier states are independent of their fractional occupation,

$$
\frac{\partial E}{\partial f_{i}}=\epsilon_{i}\left(f_{i}\right)=\epsilon_{i}, \quad f_{i} \in(0,1)
$$

where $f_{i}$ is the occupation of state $i$ with eigenvalue $\epsilon_{i}$ [see Fig. $1(\mathrm{~g})]$.

The HOMO-I condition. In DFT there is no Koopmans theorem [35], which precludes a direct assignment of the KS eigenvalues as charged excitation energies. However, in exact KS theory $I$ is equal to the negative of the HOMO [36-38]:

$$
\epsilon_{N_{0}}\left(N_{0}\right)=-I \text {. }
$$

Furthermore, Chong et al. and Grisenko et al. put forward theoretical and numerical arguments that the KS energies of occupied states can be interpreted as approximate but rather accurate vertical ionization potentials [39-42].

The derivative discontinuity. So far, $\partial E / \partial N$ has been restricted to open intervals of integers. In general, the total energy will exhibit kinks at integer electron numbers. Therefore, at integer electron numbers $\partial E / \partial N$ does not exist (unless $A$ and
$I$ are degenerate). Indeed, the limit from the right and the limit from the left

$$
\begin{aligned}
& \frac{\partial E^{-}}{\partial N}\left(N_{0}\right)=-I, \\
& \frac{\partial E^{+}}{\partial N}\left(N_{0}\right)=-A
\end{aligned}
$$

differ by the fundamental gap (i.e., $I-A$ ) [see Fig. 1(d)].

The question is what does the Kohn-Sham system for $N_{0}$ electrons capture and what is the meaning of the HOMO and LUMO states? While there is consensus that the HOMO energy of the $N_{0}$ electron system equals $-I$, the LUMO level cannot be assigned to $A$ in the exact theory [17,36-38]. The reason is the so-called derivative discontinuity of the exchangecorrelation potential $\Delta v_{\mathrm{XC}}$ [43-46]. This discontinuity is responsible for the difference between the Kohn-Sham gap and the fundamental gap

$$
\begin{aligned}
E_{\text {gap }}^{\text {fund }}:=I-A=\epsilon_{N_{0}+1}\left(N_{0}\right)-\epsilon_{N_{0}}\left(N_{0}\right)+\Delta v_{\mathrm{XC}}, \\
\Delta v_{\mathrm{XC}}:=v_{x c}^{+}(r)-v_{x c}^{-}(r), \\
v_{\mathrm{XC}}^{ \pm}:=\left.\lim _{\omega \rightarrow 0} \frac{\partial E}{\partial \rho(r)}\right|_{N_{0} \pm \omega},
\end{aligned}
$$

where $\epsilon_{N_{0}+1}\left(N_{0}\right)$ is the KS LUMO and $\epsilon_{N_{0}}\left(N_{0}\right)$ the KS HOMO of the $N_{0}$ electron system. Given that HOMO $=-I$, it follows that the LUMO does not correspond to $-A$ but rather differs from it by $\Delta v_{x c}$ [see Fig. $1(\mathrm{~g})$ ].

The deviation from straight line error. An approximate $\mathrm{XC}$ functional that obeys the piecewise linearity condition is free from the deviation from straight line error (DSLE). Conversely, a functional that violates this condition is said to suffer from the DSLE. More specifically, if the DSLE manifests itself as a convex (concave) total energy function between integer charges, then it is also called delocalization (localization) error [47-50] [see Fig. 1(b)].

We note that the DSLE is sometimes also referred to as many-electron self-interaction error [51-55]. However, we prefer the wording DSLE because in our opinion "manyelectron self-interaction error" may lead to semantic confusion with the single-electron self-interaction error [56]. It is important to distinguish between these two concepts. The definition of the single-electron self-interaction error is closely related to the Fock operator in Hartree-Fock theory. There, the Fock part cancels the interaction of a single-electron orbital with itself in the Hartree term. This concept of the one-electron self-interaction error has been adopted in DFT, where for a one-electron system the Hartree, exchange, and correlation contributions must vanish in an exact theory [56], which gave rise to the widely known self-interaction correction (SIC) by Perdew and Zunger [56-58]. The DSLE and the singleelectron self-interaction error are in general not equivalent. A counterexample is Hartree-Fock theory, which is singleelectron self-interaction free but not DSLE free [47]. During the course of this paper we will exclusively use the concept of the DSLE.

Kohn-Sham vs generalized Kohn-Sham schemes. KohnSham (KS) and generalized Kohn-Sham (GKS) schemes differ in their choice of the exchange-correlation potential. 
In Kohn-Sham theory, the exchange-correlation potential is local (i.e., multiplicative). For the inclusion of exact exchange this implies that the exchange potential derives from the optimized effective potential (OEP) method [59,60]. In generalized Kohn-Sham theory, the exact exchange potential is treated in a nonlocal fashion as in Hartree-Fock (HF) theory. GKS is a generalization of KS because it regards the exact exchange contribution as part of the reference systems rather than being part of the local effective potential. All previous statements about the KS scheme carry over to GKS [49,61] (note, however, that it has been argued that the derivative discontinuity is smaller in GKS [16]).

The HOMO and LUMO in approximate theories. In exact DFT, the LUMO cannot be assigned to $A$. However, in a recent paper Yang et al. have shown [18] that if the exchangecorrelation energy is an explicit and differentiable functional of the first-order density matrix, and if the theory is DSLE free, then

$$
\epsilon_{N_{0}+1}\left(N_{0}\right)=-A(\Delta \mathrm{SCF}),
$$

i.e., the LUMO corresponds to the negative of $A$ as obtained by total energy differences ( $\triangle \mathrm{SCF}$ ). In other words, the limit from the right of the partially filled LUMO equals the empty LUMO level [see Fig. 1(i)]. Standard semilocal functionals are differentiable, because the derivative discontinuity is absent. ${ }^{1}$ Standard hybrid functionals are also differentiable because the exact-exchange energy is and because the accompanying local potential does not exhibit a derivative discontinuity [18]. However, the OEP exact-exchange formalism is not differentiable [18]. Collecting the results for DSLE-free hybrid functionals that are differentiable in the first-order density matrix, we thus have the following:

(1) $\epsilon_{N_{0}}\left(N_{0}\right)=-I(\Delta \mathrm{SCF})$.

(2) $\epsilon_{N}\left(f_{N}\right)=$ const, $\quad f_{N} \in(0,1)$.

(3) $\epsilon_{N+1}\left(f_{N+1}\right)=$ const, $\quad f_{N+1} \in(0,1)$.

(4) $\epsilon_{N_{0}+1}\left(N_{0}\right)=-A(\triangle \mathrm{SCF})$.

Conditions 1-3 are valid for the exact $\mathrm{XC}$ functional and for approximate yet DSLE-free XC functionals. Functionals that suffer from the DSLE do not obey conditions 1-3 because the partially occupied frontier orbitals are no longer constant [see Figs. 1(e) and 1(h)]. Condition 4 holds for DSLE-free approximate functionals that are explicit and differentiable functionals of the first-order density matrix [18].

For such a class of DSLE-free approximate XC functionals, conditions 1-4 may be rewritten more compactly as

$$
\begin{gathered}
\epsilon_{N}\left(f_{N}\right)=\text { const }, \quad f_{N} \in[0,1] \\
\epsilon_{N+1}\left(f_{N+1}\right)=\text { const }, \quad f_{N+1} \in[0,1] .
\end{gathered}
$$

Notation. In the following, we will abbreviate our notation by referring to the LUMO as $\epsilon_{N+1}(f)=\epsilon^{L}(f)$ and calling it fractionally charged LUMO. Similarly for the HOMO we will write $\epsilon_{N}(f)=\epsilon^{H}(f)$, which will then be called fractionally charged HOMO level.

\footnotetext{
${ }^{1}$ This "common wisdom" has been recently challenged in the light of an ensemble generalization of the KS formalism $[66,67]$.
}

\section{EXISTING THEORETICAL APPROACHES}

Up until now, several strategies have emerged to incorporate the straight line conditions (15) and (16) in approximate DFT functionals. These can be roughly divided into three classes: the first is to restore the linearity condition for a given standard (semi)local or hybrid functional [62-64]. In the second class, the parametrization of hybrid functionals is chosen such that the straight line condition is satisfied. The third class combines hybrid functionals with the single-particle Green's function from many-body perturbation theory.

There are several approaches in the first class. They either aim to correct for those terms in the total energy that are nonlinear in the density (i.e., the Hartree and XC energy) [62,65], or introduce an ensemble generalization of the Kohn-Sham reference system [63,66-69], or add a penalty functional to the KS Hamiltonian [64,70-73], or use the DFT $+U$ approach [74]. These approaches do not change the total energy (and for applications more importantly the total energy differences) at integer charges and simultaneously restore the straight line property. This is desirable because these functionals typically perform very well for, say, $\triangle \mathrm{SCF}$ or atomization energy calculations. Simultaneously, they improve the position of the HOMO and LUMO levels in describing charged excitation energies. The main challenge of these approaches is to assure the unitary invariance of the one-body density matrix [64,75]. Furthermore, if the correction is applied in the KS schemes, then the orbital-dependent correction needs to be treated with the effective optimized potential (OEP) method in order to obtain the corresponding local KS potential. In a slightly different approach, Sadigh et al. showed recently that by enforcing the straight line condition with a simple correction, additional charge can be localized correctly in polaronic states in solids [76].

The second class of approaches is to employ hybrid functionals within the GKS framework. The basic idea is to parametrize the exact-exchange energy such that the linearity condition is restored [19-23]. This route has been predominantly followed with the long-range separated BaerNeuhauser-Livshiz (BNL) hybrid functional [24,25]. The BNL functional is based on a separation of the Coulomb potential into a long- and short-range part via the error function $r^{-1}=$ $\operatorname{erf}(\gamma r) r^{-1}+\operatorname{erfc}(\gamma r) r^{-1}$. The long-range exchange energy is then treated with exact exchange and the short-range part is approximated by the local density approximation (LDA) [77]. In this approach, the range separation parameter $\gamma$ is optimally tuned such that the HOMO-I condition [78] is fulfilled. More precisely, one seeks that value of $\gamma$ for which the GKS HOMO coincides with $-I$ obtained from a $\triangle S C F$ calculation. This is done either for the neutral $N$ electron system or additionally also for the $N+1$ electron system in order to improve the description of the fundamental gap. In contrast to the first class of self-interaction corrections, where one seeks to restore the linearity condition, one a priori requires only the HOMO-I condition, which does not necessarily imply that also the straight line behavior is obeyed. However, it has been observed that once the HOMO-I condition is fulfilled, also the straight line behavior is significantly improved for the BNL as well as for other range separated hybrid functionals [78-80]. The advantage of the second class of approaches via 
hybrid functionals is that they incorporate a system-dependent fraction of exact exchange, which is determined based on rigorous DFT arguments instead of globally fixing it or by fitting it to experiment [8]. That the fraction of exact exchange should be system dependent was argued via its relationship to the dielectric constant and has also been exploited for the construction of hybrid functionals [81-84].

The main disadvantage of the second class of approaches is that they explicitly rely on the $\triangle \mathrm{SCF}$ approach, which is difficult for periodic systems [85]. The third class of approaches therefore enforces the HOMO-I condition differently, e.g., by calculating the poles of the single-particle Green's function, which correspond to charged excitation energies and thus also the ionization energy. In this approach, the parameters of a hybrid functional are determined by minimizing the self-energy correction of the HOMO level within the $G_{0} W_{0}$ approximation [11] for the self-energy and thus the Green's function [12,15] [see Eqs. (1)-(3)]. For the PBE hybrid functional, we then denote the optimized functional $\operatorname{PBEh}\left(\alpha^{*}\right)$. The advantage of this scheme is that it is applicable to molecules and solids alike $[12,86]$. Furthermore, it may also be regarded as a plausible, albeit not unique $[87,88]$, solution to the starting point dependence of the $G_{0} W_{0}$ scheme [89-91] where the self-energy is evaluated perturbatively using DFT input orbitals and eigenvalues. The starting point dependence can be rigorously removed in the fully self-consistent $G W(\operatorname{sc} G W)$ approach [92]. However, sc $G W$ is computationally much more expensive than hybrid functionals and can currently not be applied to large systems. It should be noted that $G W$ is not an exact theory and, therefore, one cannot expect $\operatorname{PBEh}\left(\alpha^{*}\right)$ to have no DSLE. However, the remaining DSLE is vanishingly small, as we will demonstrate in the paper.

\section{COMPUTATIONAL METHOD}

All calculations reported in this work were performed with the FHI-AIMS package [93], an all-electron code that employs numeric atom-centered orbitals (NAOs) as basis functions. Methods that go beyond a semilocal DFT approximation (hybrid functionals, $G W$, RPA) employ a resolution of identity approach for the nonlocal Fock operator and for the linear density response function [94]. The basis set hierarchy in FHI-AIMS is arranged in tiers. Additionally, Gaussiantype basis sets and valence-correlation consistent are NAOs available [95,96]. Throughout this work, we employ tight integration settings [93]. We use a tier 2 basis set for hybrid functionals, unless stated otherwise. For $G W$ and rPT2, the basis set convergence is much slower than for standard DFT functionals [32,94]. For these calculations, we therefore used a much larger basis set, which will be stated in Sec. VI.

\section{EXAMPLE OF TTF, TCNQ, AND THE TTF-TCNQ COMPLEX}

In this section, we apply the principles of Sec. II to the donor-acceptor complex TTF-TCNQ and to the problem of erroneous charge transfer in the ground state. Readers that are interested in our benchmark results for atomization energies, weak interaction energies, and barrier heights may proceed straight to Sec. VI.

\section{A. Individual molecules of TTF and TCNQ}

We illustrate the DSLE for the TCNQ and TTF molecules. Figure 3(a) shows the total energy as a function of partial
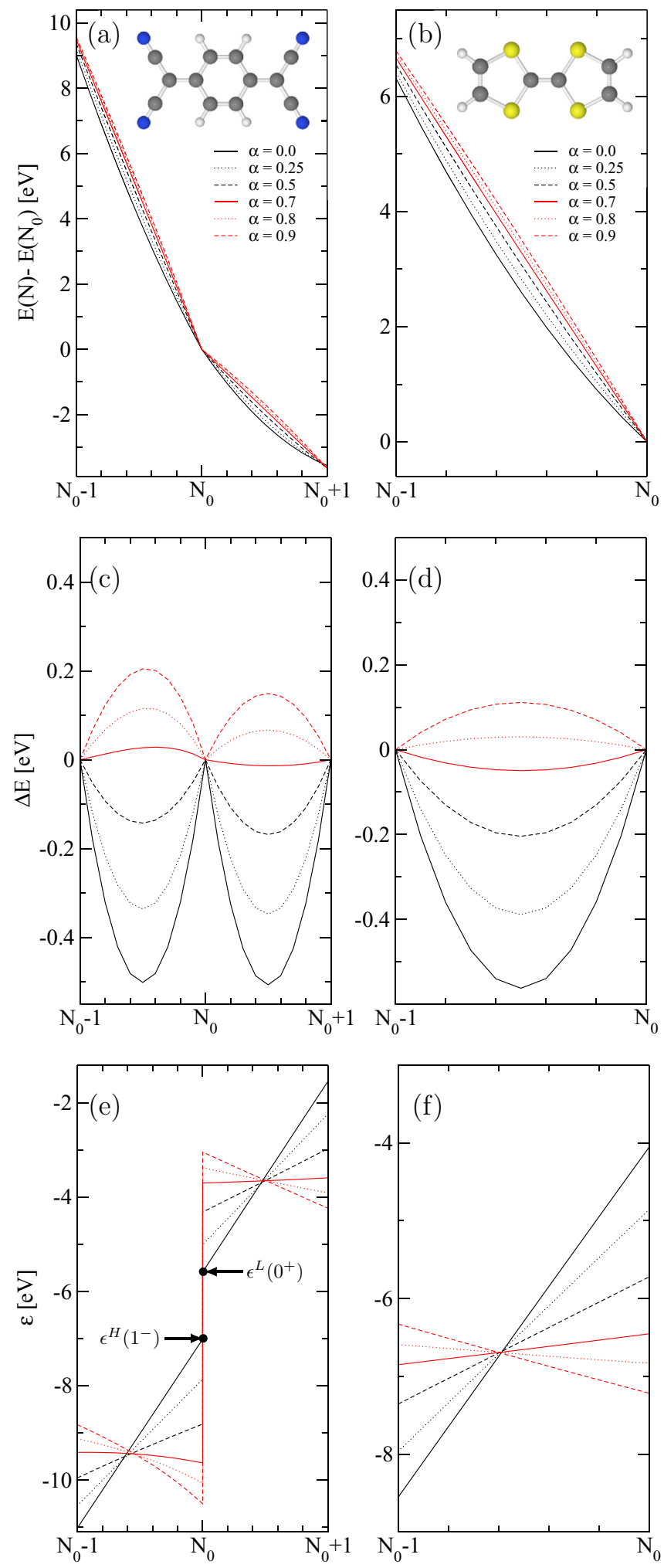

FIG. 3. Total energy for the TCNQ (a) and TTF (b) molecules as a function of partial occupation. (c), (d) Show the deviation from the straight line behavior; (e), (f) show GKS eigenvalues at partial occupation. 
occupation for the TCNQ molecule with respect to the neutral molecule. The same plot for TTF is shown in Fig. 3(b). However, since TTF is an electron donor, it does not bind an excess electron and therefore we will only consider partial electron removal.

To make the deviation from the straight line condition more visible, we define

$$
\Delta E(N):= \begin{cases}E(N)-I\left(N_{0}-N\right)-E\left(N_{0}\right), & N \leqslant N_{0} \\ E(N)-A\left(N_{0}-N\right)-E\left(N_{0}\right), & N>N_{0}\end{cases}
$$

on the interval $\left[N_{0}-1, N_{0}+1\right]$. This function is a measure for the deviation of the straight line behavior with slopes obtained by the $\triangle$ SCF approach. It is by construction zero at integer values and the sign of $\Delta E(N)$ is chosen such that a negative value corresponds to a convex total energy curve, i.e., $E(N)$ lies below a straight line.

Figure 3(c) shows $\Delta E(N)$ for the TCNQ molecule. Low $\alpha$ functionals significantly deviate from the straight line condition. In particular for $\alpha \leqslant 0.5$ the total energy is a convex function of electron number and thus exhibits a delocalization error. In contrast, for $\alpha \geqslant 0.8$ the total energy is concave and thus exhibits a localization error. For $\alpha=0.7$, the straight line condition is almost obeyed, although there is a small residual localization error upon partial electron removal and delocalization error upon partial electron addition. Turning to TTF, shown in Fig. 3(d), the overall behavior is similar. However, the $\alpha$ value for which the deviation from linearity condition is minimized is now at a slightly larger value of about 0.8. Again, this value gives an almost straight line feature for both the partially occupied HOMO and LUMO levels. Hence, in agreement with Ref. [19] we conclude that the linearity condition is best obeyed for large $\alpha$ values ( 0.7 for TCNQ and 0.8 for TTF). In contrast, the standard values $\alpha=0$ or $\frac{1}{4}$ which correspond to the PBE and PBE0 functionals significantly violate the straight line condition.

The DSLE immediately translates into the eigenvalues of the frontier orbitals. Figures 3(e) and 3(f) show the eigenvalues as function of occupation:

$$
\begin{array}{ll}
\epsilon^{H}(f), & N_{0}-1 \leqslant N \leqslant N_{0}, \quad f=N-N_{0}+1 \\
\epsilon^{L}(f), & N_{0} \leqslant N \leqslant N_{0}+1, \quad f=N-N_{0} .
\end{array}
$$

It can be seen that the choice of $\alpha$ that minimizes the deviation from the straight line behavior gives constant eigenvalues for the frontier orbitals. This is to be expected because Janak's theorem dictates

$$
\frac{\partial E}{\partial f}= \begin{cases}\epsilon^{H}(f), & N_{0}-1<N<N_{0} \\ \epsilon^{L}(f), & N_{0}<N<N_{0}+1\end{cases}
$$

which is constant if the total energy is piecewise linear. In this case, the eigenvalues also correspond to the negative of $I$ and $A$ as given by the $\triangle \mathrm{SCF}$ approach

$$
\frac{\partial E}{\partial f}= \begin{cases}\epsilon^{H}(f)=\mathrm{const}=-I(\Delta \mathrm{SCF}), & N_{0}-1<N<N_{0} \\ \epsilon^{L}(f)=\mathrm{const}=-A(\Delta \mathrm{SCF}), & N_{0}<N<N_{0}+1 .\end{cases}
$$

For other $\alpha$ values, the eigenvalues depend on the occupation and their variation can be as large as several $\mathrm{eV}$ in the extreme case of $\alpha=0$, which is equivalent to the PBE functional.
Furthermore, for any choice of $\alpha$, the slope of the eigenvalues appears to be constant. It gradually increases from being positive $(\alpha=0.0)$ to zero $(\alpha \sim 0.7)$ and negative for $\alpha>0.7$. This is a consequence of the curvature of the total energy. For small $\alpha$ values, the total energy is convex. Therefore, its derivative monotonically increases. For large $\alpha$ values, the total energy is concave which results in a monotonically decreasing first derivative. If the total energy is piecewise linear, then its curvature is zero and therefore its slope is zero between integer charges.

While the slope of the eigenvalues strongly depends on $\alpha$, Fig. 3(e) also shows that all curves cross at a point very close to $N_{0} \pm 0.5$, i.e., at half occupation of the HOMO and LUMO, respectively. In particular, they also cross the constant eigenvalue lines for which the eigenvalues correspond to the negative of $I$ and $A$, respectively. This implies that the SlaterJanak transition state [97-99], which obtains $I$ and $A$ by the eigenvalue of the half-filled HOMO and LUMO levels,

$$
\begin{aligned}
& I \approx-\epsilon^{H}(0.5), \\
& A \approx-\epsilon^{L}(0.5),
\end{aligned}
$$

is a valid approximation, independent of $\alpha$. In the following, we argue that this approximation is sensible if the total energy is, to a good approximation, a quadratic function of the particle number. The Slater-Janak transition state may be obtained by integrating Janak's theorem. For the ionization potential, this gives

$$
-I=\int_{0}^{1} \epsilon^{H}(f)=e^{H}(\xi),
$$

where in the last step the mean value theorem of calculus was used, that guarantees a $\xi \in[0,1]$ such that the integral may be obtained by the HOMO eigenvalue with certain occupation $\xi$. While this is still exact, the Slater-Janak transition state makes the approximation $\xi \approx 0.5$. Now, assume that the total energy is a quadratic function of $f$ :

$$
\begin{gathered}
E(f)=E_{0}+f E_{0}^{\prime}+f^{2} E_{0}^{\prime \prime}, \\
\epsilon^{H}(f)=E_{0}^{\prime}+2 f E_{0}^{\prime \prime} .
\end{gathered}
$$

With the left-hand side of Eq. (22), we have $-I=E_{0}^{\prime}+$ $E_{0}^{\prime \prime}=\epsilon^{H}(0.5)$. An analogous argument can be given for the electron affinity. Thus, the closer the total energy follows a quadratic behavior, the better the Slater-Janak transition state will perform. That in standard DFT approximations the Slater-Janak transition state is a good approximation is not very surprising because the Hartree energy, which is quadratic in $f$, gives a large contribution to the total energy. Also, note that Eq. (24) implies that the eigenvalues are linear as a function of occupation, with slopes that are given by the curvature $E^{\prime \prime}$. This is in agreement with Figs. 3(e) and 3(f). Note that Eqs. (23) and (24) contain the special case where $E(f)$ is piecewise linear by setting $E_{0}^{\prime \prime}=0$.

From this perspective, the results of Fig. 3 appear as different sides of the same coin: for low $\alpha$ values the total energy is convex $E_{0}^{\prime \prime}>0$ and thus the slope of the eigenvalues is positive and constant. For straight line segments, the curvature of each segment is zero $E^{\prime \prime}=0$ (at $\alpha \sim 0.7$ ), 
implying constant eigenvalues. For very large $\alpha$ values, the total energy is convex $E_{0}^{\prime \prime}<0$, which results in a negative but constant slope of the eigenvalues. On the other hand, the Slater-Janak transition state remains accurate for any choice of $\alpha$. We stress that in the DSLE-free case, the Slater-Janak transition state is trivial because then the eigenvalues of the frontier orbitals are independent of their occupation.

Having clarified the connection between the linearity condition, the GKS eigenvalues at partial occupation, and the Slater-Janak transition state, we now turn to the eigenvalues at integer particle number. We first observe from Fig. 3(e) that for all $\alpha$ values, the HOMO and LUMO of the neutral molecules correspond to the one-sided limits

$$
\begin{gathered}
\epsilon^{H}(1)=\lim _{f \rightarrow 1^{-}} \epsilon^{H}(f), \\
\epsilon^{L}(0)=\lim _{f \rightarrow 0^{+}} \epsilon^{L}(f),
\end{gathered}
$$

i.e., the fully occupied HOMO corresponds to the limit from the left and the empty LUMO corresponds to the limit from the right (illustrated for $\alpha=0$ by the arrows). The fact that the derivative of the total energy agrees with the eigenvalues for both the HOMO and the LUMO demonstrates that the PBE hybrid family is explicit and differentiable in the first-order density matrix, i.e., it lacks the derivative discontinuity [cf. Fig. 1(e)].

However, the actual quality of the HOMO and LUMO eigenvalues strongly depends on the DSLE and thus on $\alpha$. Indeed, the HOMO and LUMO values at $N_{0}$ vary by more than $2 \mathrm{eV}$ as a function of $\alpha$. In particular, for low $\alpha$ values, where the slope of the eigenvalues is positive (and hence, the total energy is convex) $\epsilon^{H}(1)>\epsilon^{H}(0.5)$ or, equivalently, $\epsilon^{H}(1)>-I$. For very large $\alpha$ values, the slope of the eigenvalues is negative (and hence, the total energy is concave) $\epsilon^{H}(1)<\epsilon^{H}(0.5)$. Therefore, $\epsilon^{H}(1)<-I$. Finally, if the total energy is DSLE free, then $\epsilon^{H}(1)=\epsilon^{H}(0.5)=-I$. In summary, the situation for the HOMO is

$$
\epsilon^{H}(1) \begin{cases}>-I(\triangle \mathrm{SCF}), & E \text { convex } \\ =-I(\Delta \mathrm{SCF}), & E \text { pw. linear } \\ <-I(\Delta \mathrm{SCF}), & E \text { concave }\end{cases}
$$

and similarly for the LUMO

$$
\epsilon^{L}(0) \begin{cases}<-A(\triangle \mathrm{SCF}), & E \text { convex } \\ =-A(\Delta \mathrm{SCF}), & E \text { pw. linear } \\ >-A(\Delta \mathrm{SCF}), & E \text { concave. }\end{cases}
$$

As a consequence, the fundamental gap $A-I$ is underestimated for a convex total energy functional (delocalization error) and overestimated for a concave total energy functional (localization error). And, if the total energy is piecewise linear, both the HOMO and LUMO levels correspond to the negative of the $I$ and $A$ as obtained by the $\triangle$ SCF approach. Eventually, also the HOMO-LUMO gap corresponds to the fundamental gap. However, this does not imply that $I$ and $A$ are exact because the straight line behavior does not guarantee that the end points [i.e., $E\left(N_{0}\right)$ and $\left.E\left(N_{0} \pm 1\right)\right]$ are correct.
TABLE I. Optimized $\alpha$ values obtained by requiring the HOMO$I$ condition via the $\triangle \mathrm{SCF}$ and $G_{0} W_{0}$ approach.

\begin{tabular}{lcc}
\hline \hline & $\Delta S C F$ & $G_{0} W_{0}$ \\
\hline TCNQ & 0.66 & 0.79 \\
TTF & 0.77 & 0.76 \\
\hline \hline
\end{tabular}

\section{B. Comparison of methods to determine the optimum $\alpha$ value}

Having illustrated the overall dependence on $\alpha$, we now compare two different methods to obtain $\alpha$ : enforcing the straight line condition and the $G_{0} W_{0}$ scheme [i.e., the $\operatorname{PBEh}\left(\alpha^{*}\right)$ functional]. The $\alpha$ values of the $G_{0} W_{0}$ scheme have previously been reported in Ref. [12] and are listed in Table I. For both TCNQ and TTF, they exceed 0.75 and are thus significantly larger than the $\alpha$ range of $0.1-0.3$ found for solids with the same scheme [86]. These different $\alpha^{*}$ values may be rationalized by the inverse relation between $\alpha$ and the dielectric constant [82]. Small molecules have a dielectric constant close to one, and therefore their $\alpha^{*}$ value is close to one, too. For solids, the dielectric constant is larger, which eventually yields a smaller $\alpha^{*}$ value [86].

The DSLE-free $\alpha$ values obtained in this work with the HOMO-I $\triangle \mathrm{SCF}$ scheme are also reported in Table I. For TTF, the $G_{0} W_{0}$ approach gives virtually the same $\alpha$ value as the $\triangle \mathrm{SCF}$ approach and a slightly larger value for TCNQ. Thus, the $\operatorname{PBEh}\left(\alpha^{*}\right)$ functional significantly reduces the DSLE and only exhibits a small tendency towards a residual localization error (concave behavior). This is a rather remarkable feature of the $G_{0} W_{0}$ self-energy, given that it is the simplest nontrivial self-energy in Hedin's equations [11,100].

\section{TTF-TCNQ complex}

So far, we have shown that the DSLE is responsible for the deviation of the GKS frontier levels from $I(\triangle \mathrm{SCF})$ and $A(\triangle \mathrm{SCF})$. In practical calculations, this does not pose any mayor problem because one can always resort to a $\triangle \mathrm{SCF}$ calculation or use the Slater-Janak transition state to determine these values. However, in the following, we will show that the DSLE may lead to an unphysical ground-state density. To this end, we consider the complex that is built from TTF and TCNQ. We study vertical electron transfer from TTF to TCNQ, i.e., we neglect effects from geometry relaxation in the transfer process.

We start with the noninteracting case, in which the two molecules are infinitely separated (sketched in Fig. 4). We define a transfer energy as

$$
\begin{aligned}
E_{t}(\omega):= & E_{\mathrm{TCNQ}}\left(N_{0}+\omega\right)+E_{\mathrm{TCNQ}}\left(N_{0}-\omega\right) \\
& -\left\{E_{\mathrm{TCNQ}}\left(N_{0}\right)+E_{\mathrm{TCNQ}}\left(N_{0}\right)\right\} .
\end{aligned}
$$

$E_{t}$ is an energetic measure for (partial) $\omega$ electron transfer with respect to the overall neutral molecules with $N_{0}$ electrons (note that both neutral molecules have 104 electrons and therefore share a common $N_{0}$ ). For the exact $\mathrm{XC}$ functional, $E_{\mathrm{TCNQ}}\left(N_{0} \pm \omega\right)$ and $E_{\mathrm{TTF}}\left(N_{0} \pm \omega\right)$ is piecewise linear. Therefore, also $E_{t}(\omega)$ is piecewise linear. Using Janak's theorem, 

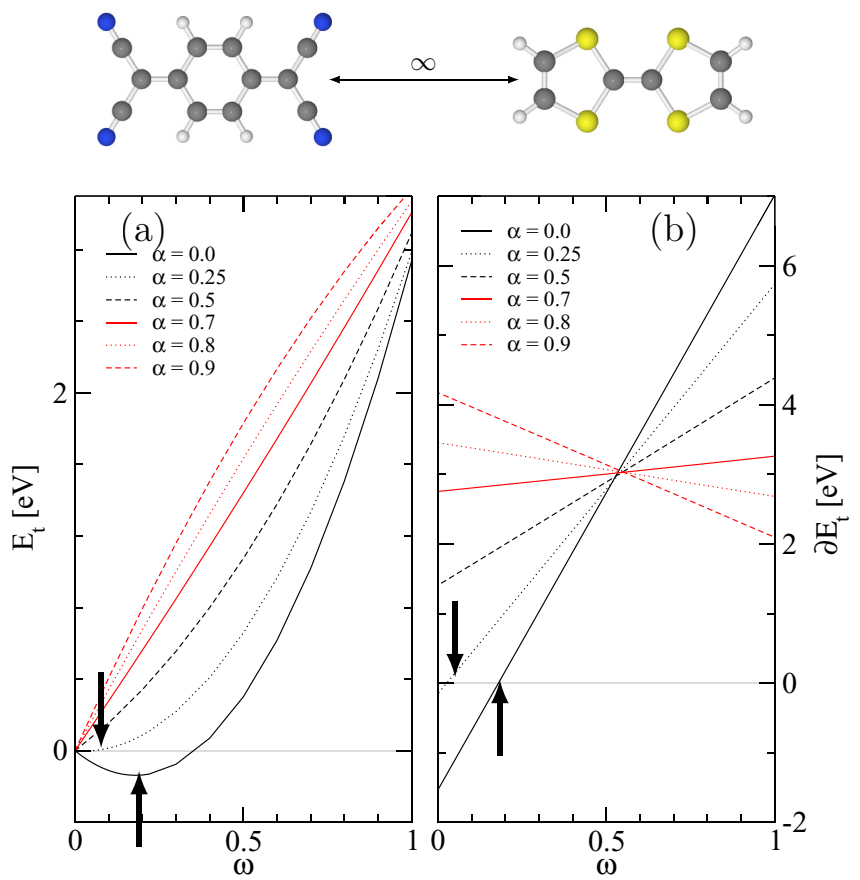

FIG. 4. Transfer energy (a) and its derivative (b) for the TTFTCNQ complex.

the derivative of $E_{t}$ is given by

$$
\frac{\partial E_{t}}{\partial \omega}= \begin{cases}\epsilon_{\mathrm{TCNQ}}^{L}(\omega)-\epsilon_{\mathrm{TTF}}^{H}(1-\omega), & 0<\omega<1 \\ \epsilon_{\mathrm{TTF}}^{L}(\omega)-\epsilon_{\mathrm{TCNQ}}^{H}(1-\omega), & -1<\omega<0\end{cases}
$$

where we have used the chain rule and expressed the occupation number of the HOMO $f^{H}=1-\omega$ and LUMO $f^{L}=\omega$ in terms of $\omega$. Thus, the slope of $E_{t}$ is given by the difference between the partially occupied TCNQ-LUMO level and TTF-HOMO level if partial electrons are transferred from TTF to TCNQ (and conversely if electrons are transferred from TCNQ to TTF). Using Eq. (6), Eq. (30) may be rewritten for a DSLE-free functional as

$$
\frac{\partial E_{t}}{\partial \omega}= \begin{cases}I_{\mathrm{TTF}}-A_{\mathrm{TCNQ}}, & 0<\omega<1 \\ I_{\mathrm{TCNQ}}-A_{\mathrm{TTF}}, & -1<\omega<0 .\end{cases}
$$

Hence, the slope of $E_{t}$ is given by the difference of $I$ and $A$ independent of the amount of transferred charge for the exact functional. In order to facilitate our discussion, we focus on the process where partial electrons are transferred from TTF to TCNQ (the reverse process is unphysical because TTF does not bind an additional electron).

For the exact functional that is piecewise linear $\partial E_{t} / \partial \omega$ is constant on the open interval because $E_{t}$ is a linear combination of piecewise linear functions. For approximate functionals that lack the derivative discontinuity and are DSLE free, the one-sided limits are given by the difference of the neutral GKS eigenvalues

$$
\begin{aligned}
\frac{\partial E_{t}}{\partial \omega}(0) & =\epsilon_{\mathrm{TCNQ}}^{L}(0)-\epsilon_{\mathrm{TTF}}^{H}(1) \\
& =\frac{\partial E_{t}}{\partial \omega}(\omega)=\epsilon_{\mathrm{TCNQ}}^{L}(\omega)-\epsilon_{\mathrm{TTF}}^{H}(1-\omega) \\
& =\text { const }, \quad \omega \in(0,1) .
\end{aligned}
$$

Recall that this is only true for approximate functionals that are piecewise linear and lack the derivative discontinuity. Indeed, for the exact functional the first equality sign is not true because there are contributions from the derivative discontinuity upon taking the limit $\omega \rightarrow 0^{+}$. The third and fourth equality signs, however, hold true because the exact functional is piecewise linear. For approximate functionals that are not piecewise linear, Eq. (32) is also not true because they violate the constancy requirement

$$
\frac{\partial E_{t}}{\partial \omega}(\omega)=\epsilon_{\mathrm{TCNQ}}^{L}(\omega)-\epsilon_{\mathrm{TTF}}^{H}(1-\omega) \neq \text { const }, \quad \omega \in(0,1) .
$$

This can be seen in Fig. 4, which reports in (a) the transfer energy and in (b) its derivative as a function of transferred electrons $\omega$ for various $\alpha$ values. For low $\alpha$ values, the transfer energy is concave. Therefore, its derivative is not constant. Increasing $\alpha$ gradually reduces the convexity of $E_{t}$ and the slope of its derivative reduces. At $\alpha \approx 0.7$, the transfer energy is close to piecewise linear, which results into an almost constant difference in the eigenvalues, irrespective of transferred electrons. Therefore, condition (32) is met for $\alpha \approx 0.7$. For $\alpha \geqslant 0.8$, the transfer energy is concave, which results in a negative slope in its derivative.

The convexity of $E_{t}$ for low $\alpha$ values has important consequences for charge transfer. Recall that $E_{t}$ is an energetic measure for charge transfer in the asymptotic limit. Now, for $\alpha=0$ (PBE) and $\alpha=\frac{1}{4}$ (PBE0), $E_{t}$ crosses the zero line in Fig. 4. This implies that the system gains energy upon partial electron transfer even if the two molecules are infinitely far away. Electrons will be transferred until $E_{t}$ reaches its minimum as indicated by the black arrows in Fig. 4(a). The minimum is reached when $E_{t}$ has zero slope [black arrows in Fig. 4(b)]. From Eq. (33), the slope is zero for a specific $\omega^{\infty}$ for which

$$
\epsilon_{\mathrm{TCNQ}}^{L}\left(\omega^{\infty}\right)-\epsilon_{\mathrm{TTF}}^{H}\left(1-\omega^{\infty}\right)=0 .
$$

Thus, in the noninteracting limit, partial electrons will be transferred from the TTF-HOMO level into the TCNQLUMO level until both partially occupied orbitals align [54]. Numerically, this amounts to $\omega^{\infty} \approx 0.2$ (PBE) and $\omega^{\infty} \approx 0.05$ (PBE0) transferred electrons. These are qualitative errors that arise from the convexity. More precisely, the convexity must be large enough such that the zero line is crossed. For example, for $\alpha=0.5, E_{t}$ is still convex (positive slope of $\partial E_{t} / \partial \omega$ ), but it does not cross the zero line and thus does not exhibit asymptotic charge transfer. Furthermore, also functionals that obey piecewise linearity or are concave to not exhibit this artifact because they have their minimum at zero charge transfer.

The definition of $E_{t}$ and thus its physical implications only give access to the noninteracting limit. In principle, this picture breaks down if two molecules approach each other and eventually start to interact. Then, additional effects such as hybridization or Coulomb stabilization between the transferred electron and the remaining hole occur, which are not captured in this simple picture. However, in the specific case of TTF and TCNQ, the interaction is weak in the binding regime. Therefore, conclusions from the asymptotic limit carry over to 

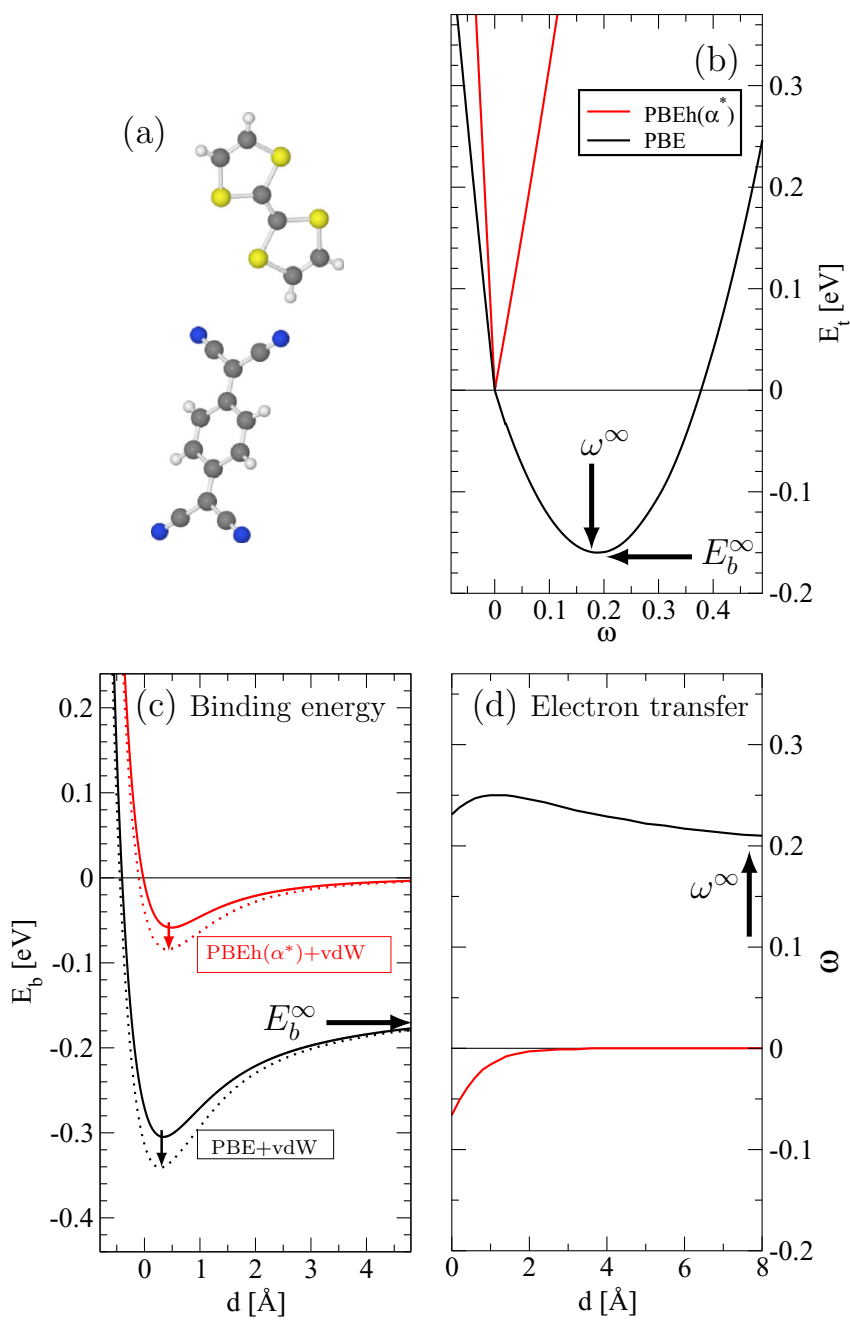

FIG. 5. Binding energy (c) and Hirshfeld charge transfer (d) for the TTF-TCNQ complex as a function of distance. A sketch of the geometry is shown in (a) which was obtained by cutting out a dimer of the TTF-TCNQ interface. The reference distance $d=0$ corresponds to the equilibrium geometry the dimer would have in the periodic interface. (b) Shows a zoom of the transfer energy.

the TTF-TCNQ complex. We take as illustrative example the $\operatorname{PBE}(\alpha=0)$ and the $\operatorname{PBEh}\left(\alpha^{*}\right)$ functionals, where $\alpha^{*} \approx 0.8$.

Figure 5(c) shows the binding energy and the electron transfer [Fig. 5(d)] as a function of the TTF-TCNQ distance.

The electron transfer was calculated by means of a Hirshfeld analysis [101]. The geometry [sketch in Fig. 5(a)] was obtained by cutting out a dimer of a periodic TTF-TCNQ interface. The reference distance $d=0$ corresponds to the equilibrium distance of the TTF-TCNQ interface obtained with the van der Waals corrected PBE functional (PBE+vdW).

Both the binding curve and charge transfer curve are very similar in shape but significantly differ in their offset. The PBE binding energy does not decay to zero for large intermolecular distance, but rather approaches a constant negative value. This value is also given by the $y$ minimum of the transfer energy as illustrated in Fig. 5(b). The $\operatorname{PBEh}\left(\alpha^{*}\right)$ functional is almost DSLE free. As a consequence, the minimum of $E_{t}$ is located at the origin. Therefore, the binding energy of $\operatorname{PBEh}\left(\alpha^{*}\right)$ approaches zero in the large-separation limit.
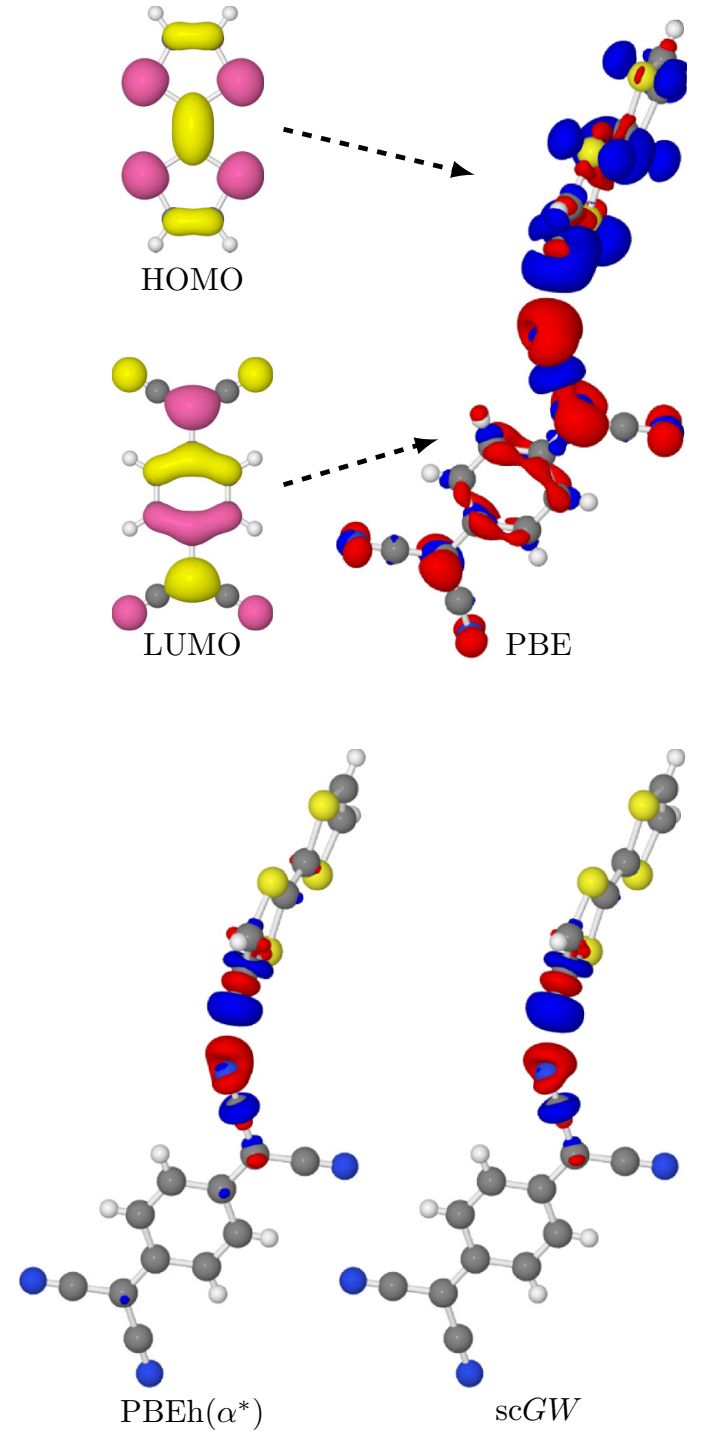

FIG. 6. Charge density difference $\delta \rho=\rho$ (dimer) $-\rho$ (TTF) $\rho$ (TCNQ) for $\operatorname{PBE}, \operatorname{PBEh}\left(\alpha^{*}\right)$, and fully self-consistent $G W(\operatorname{sc} G W)$. Also shown are the TTF-HOMO and TCNQ-LUMO orbitals. Blue corresponds to electron density depletion and red corresponds to electron density accumulation. The yellow and pink colors for the orbitals label the sign of the orbital.

The electron transfer in PBE does not decay to zero, as indicated by the black arrow in Fig. 5(d). In fact, at very large intermolecular distance, the electron transfer is given by the minimum of $E_{t}$ as shown in Fig. 5(b). Near the equilibrium distance, the electron transfer amounts to about 0.25 electrons. In contrast, the predicted electron transfer of $\operatorname{PBEh}\left(\alpha^{*}\right)$ decays to zero, as predicted by $E_{t}$. Only near the equilibrium distance $\operatorname{PBEh}\left(\alpha^{*}\right)$ predicts a very small electron redistribution.

The difference between the ground-state density of PBE and $\operatorname{PBEh}(\alpha)$ at the equilibrium geometry $(d=0)$ can be also seen in the charge density difference $\delta \rho=\rho$ (dimer) $-\rho$ (TTF) $\rho$ (TCNQ). It is plotted in Fig. 6. $\delta \rho$ qualitatively differs between $\operatorname{PBE}$ and $\operatorname{PBEh}\left(\alpha^{*}\right)$. PBE predicts electron transfer from a spatial distribution that corresponds to the TTF HOMO to the TCNQ LUMO. This behavior of PBE can already be anticipated from the aforementioned discussion of the 
asymptotic limit and, in particular, from Eq. (34). In contrast, $\operatorname{PBEh}\left(\alpha^{*}\right)$ only a predicts minor electron redistribution in an area where the two molecules are close. This feature is also supported by fully self-consistent $G W(\operatorname{sc} G W)$, which allows to calculate the ground-state density on the $G W$ level of theory $[13,92,102]$.

We conclude that the convexity error induces artifacts in the description of the binding energy and charge transfer. The error occurs for small $\alpha$ values for which the system might gain energy upon interaction-free charge transfer. This erroneous behavior may be remedied by larger $\alpha$ values that give nearly DSLE-free functionals. While such high $\alpha$ values are unconventional, the question arises if this functional parametrization is still able to predict total energy differences as accurately as the well-established PBE0 functional. In particular for weakly interacting molecules, one needs to take into account van der Waals (vdW) interactions. This is also shown in Fig. 5(c). It can be seen that the inclusion of vdW interactions via the Tkatchenko-Scheffler (TS) scheme enhances the binding well by several tens of meV. For PBE, the binding curve artifact is further enhanced upon inclusion of vdW because of the wrong asymptotics. For $\operatorname{PBEh}\left(\alpha^{*}\right)$, the question remains if the method is compatible with the choice of high $\alpha$ values. In the subsequent section, we will clarify this question by means of a comparative assessment for benchmark sets.

\section{BENCHMARK RESULTS}

We will assess the performance of the $\operatorname{PBEh}\left(\alpha^{*}\right)$ functional for weak interaction energies, atomization energies, and barrier heights. Since the PBE hybrid family does not include the long-range vdW tail, we will also study two methods that take them into account: the TS scheme [30] and renormalized second-order perturbation theory (rPT2) [31,32].

The TS scheme adds the long-range vdW tail to a given approximate DFT functional (in our case a member of the PBE hybrid family)

$$
\begin{gathered}
E=E_{\text {approx }}^{\mathrm{DFT}}+E_{\mathrm{vdW}}, \\
E_{\mathrm{vdW}}=-\sum_{a, b<a} f_{\mathrm{damp}} \frac{C_{6 a b}[\rho]}{\left|R_{a}-R_{b}\right|^{6}},
\end{gathered}
$$

with $R_{a}$ being the position of atom $a$, the damping function $f_{\text {damp }}$ and the isotropic and density-dependent $C_{6 a b}[\rho]$ term that describes the vdW interactions between atoms $a$ and $b$ within a molecule. The damping function is of the form of an inverse Fermi-Dirac distribution

$$
f_{\text {damp }}=\left\{1+\exp \left[-d\left(\frac{\left|R_{a}-R_{b}\right|}{s_{R}\left(R_{a}^{0}[\rho]+R_{b}^{0}[\rho]\right)}-1\right)\right]\right\}^{-1},
$$

where $R_{a}^{0}$ is the vdW radius of atom $a, d$ is a fixed parameter, and $s_{R}$ is a range separation parameter. The damping function contains the empirical part of the TS theory. It couples the DFT functional to the vdW tail and damps away the $1 / R^{6}$ divergence at close interatomic distances. The most important empirical ingredient in the damping function is the $s_{R}$ parameter, which determines the onset of $f_{\text {damp. }}$. In the TS recipe, it is obtained by a fit for the S22 test set [103,104], that contains 22 weakly interacting molecules consisting of a few, light elements. The reference data in S22 were obtained with the coupled cluster singles, doubles, and perturbative triples [CCSD(T)] approach, that corresponds to the gold standard for weak interactions.

Alternatively, vdW interactions are automatically included by methods based on the random-phase approximation (RPA), in particular in $\mathrm{rPT} 2$ [31,32]. The advantage of this approach is that it includes vdW interactions in a seamless fashion in the sense that it does not employ an ad hoc damping function (in contrast to the TS scheme). Furthermore, rPT2 is a promising candidate for an accurate general purpose method as it has been shown to give the "most balanced" performance over a range of different electronic and chemical environments. A distinct disadvantage of rPT2 is the significant computational cost, which currently hinders its application to large systems. In rPT2, the correlation energy is obtained by adding renormalized single excitations (rSE) and second-order screened exchange (SOSEX) to RPA [31,32]:

$$
\mathrm{rPT} 2=\mathrm{RPA}+\mathrm{rSE}+\mathrm{SOSEX} .
$$

The total energy is then given by

$$
\begin{gathered}
E=\mathrm{EX}+\mathrm{rPT} 2, \\
\mathrm{EX}=T+E_{H}+E_{X}^{\mathrm{EX}}+E_{\mathrm{ext}},
\end{gathered}
$$

where EX is the exchange-only total energy. It consists of the kinetic energy $T$, the Hartree energy $E_{H}$, the exact(Fock) exchange energy $E_{X}^{\mathrm{EX}}$, and the external potential energy $E_{\text {ext }}$. Since rPT2 resides in the framework of many-body perturbation theory, it depends on the underlying functional. This means that Eq. (38) and $E_{X}^{\mathrm{EX}}$ are evaluated for DFT input orbitals. This starting point dependence is denoted "rPT2@orbital" in the following. For example, rPT2@PBE means that the rPT2 energy is evaluated for PBE input orbitals. Up until now, rPT2 calculations have been only reported for the PBE starting point in the literature [32]. Therefore, we additionally present the $\operatorname{PBE} 0$ and $\operatorname{PBEh}\left(\alpha^{*}\right)$ starting point in order to clarify the starting point dependence of rPT2 in general and the compatibility with $\operatorname{PBEh}\left(\alpha^{*}\right)$ in particular.

\section{A. Weak interaction energies}

We start with the S66 test set by Hobza and co-workers [26]. It consists of 66 molecular complexes with $\operatorname{CCSD}(\mathrm{T})$ reference interaction energies. S66 contains most common types of weak interactions, i.e., hydrogen bonds, dispersion bonds, and mixed types of bonds.

The mean absolute percentage error (MAPE) for the TSvdW scheme is presented in Fig. 7(a) for the underlying $\operatorname{PBE}(\alpha=0), \operatorname{PBE} 0\left(\alpha=\frac{1}{4}\right)$, and $\operatorname{PBEh}\left(\alpha^{*}\right)$ functionals. Figure 7(b) shows the rPT2 results for the PBE, PBE0, and $\operatorname{PBEh}\left(\alpha^{*}\right)$ starting points. The results for the TS-vdW scheme were obtained using a tier 3 FHI-AIMS basis set. The rPT2 results were obtained, following Ren et al. [31,32], for the tier 4 basis set augmented with diffuse functions from AUGCC-PV5Z [95] together with a counterpoise correction to account for the basis set superposition error [105]. S66 benchmark results for the TS scheme were already reported in the literature for PBE and PBE0 as well as for an optimally tuned range separated 


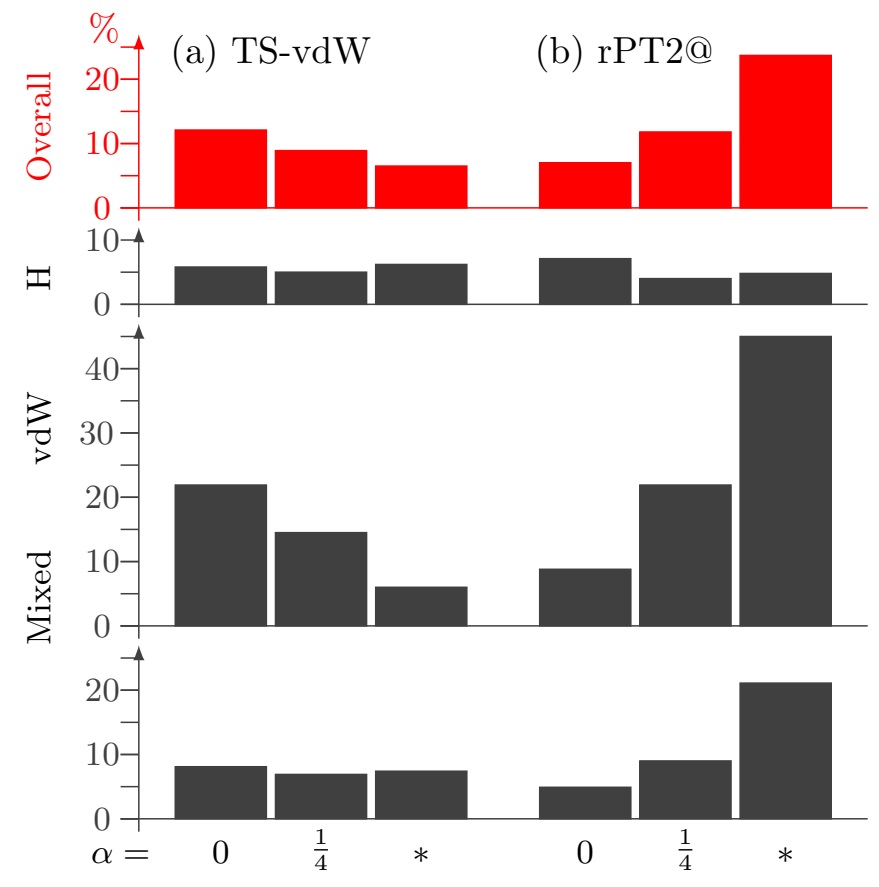

FIG. 7. Mean absolute percentage error (\%) of the (a) TS-vdW scheme [30] and (b) rPT2 [32] for the S66 test set. The errors are relative to $\operatorname{CCSD}(\mathrm{T})$ reference data [26].

hybrid functional [106-108]. rPT2@PBE results have already been reported by Ren et al. [31,32].

It can be seen that the performance of both the TS scheme and rPT2 depends on the underlying functional. In the case of the TS scheme, the accuracy improves for $\operatorname{PBEh}\left(\alpha^{*}\right)$. In particular for $\mathrm{vdW}$ interactions, for which the theory is designed, the MAPE decreases from about $22 \%$ for PBE to about $6 \%$ for $\operatorname{PBEh}\left(\alpha^{*}\right)\left(\alpha^{*} \sim 0.7-0.8\right.$, depending on the system). In the case of rPT2, the opposite behaviors occur. While only hydrogen bonds slightly benefit from a high $\alpha$ treatment, the description of $\mathrm{vdW}$ and mixed interactions degrades considerably. For these types of interactions, already the PBE0 starting point, which employs a moderate $\alpha=\frac{1}{4}$ value, clearly gives worse results than the PBE starting point.

In short, the TS scheme benefits from $\operatorname{PBEh}\left(\alpha^{*}\right)$, whereas rPT2 exhibits a clear trend of performance loss for $\alpha \neq 0$. In the subsequent discussion, we will analyze this observation for the TS scheme and rPT2, respectively.

\section{TS method}

We consider the binding curve of the pentane dimer (item number 34 in the S66 test set), for which PBE+TS exhibits the largest deviation with respect to $\operatorname{CCSD}(\mathrm{T})$. The binding curve is plotted in Fig. 8. The geometries and $\operatorname{CCSD}(\mathrm{T})$ reference data were taken from the $\mathbf{S} 66 \times 8$ set [26]. The $\mathbf{S} 66 \times 8$ data set contains 8 different intermolecular separations for each element of the S66 set. These are set by scaling the equilibrium distance $d_{0}$ by a factor of $d / d_{0}=0.9,0.95, \ldots, 2$.

Figure 8(a) shows that PBE+TS overbinds by about $60 \mathrm{meV}$ near the equilibrium distance $\left(d / d_{0} \sim 1\right)$. While all TS corrected functionals predict slightly too large equilibrium distances, the binding curves are shifted towards the $\operatorname{CCSD}(\mathrm{T})$
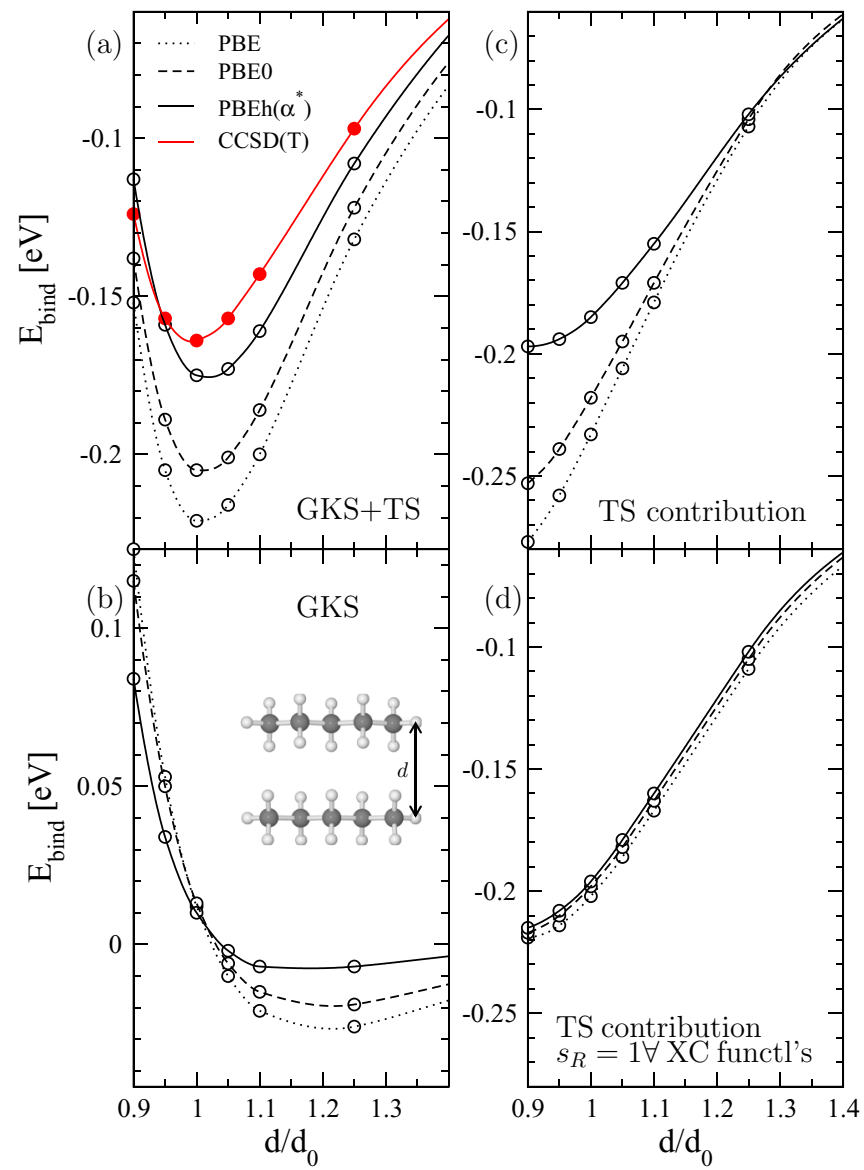

FIG. 8. Binding curves for the pentane dimer (illustrated in the inset) obtained by the TS method (a), and its contributions from the (generalized) Kohn-Sham (G)KS functionals (b), the TS-vdW tail (c), and the TS-vdW tail where the $s_{R}$ parameter is set to one (d). The binding distance is given by the scaling parameter $d$ with respect to the equilibrium distance. The $\operatorname{CCSD}(\mathrm{T})$ reference curve was taken from Ref. [26].

reference curve upon increasing the fraction of exact exchange $\left(\alpha^{*}=0.78\right.$ for the pentane dimer). As a consequence, the $\operatorname{PBEh}\left(\alpha^{*}\right)+\mathrm{TS}$ binding curve is close to the reference curve.

Tracing the reason for this observation, Figs. 8(b) and 8(c) show the binding contribution from the (G)KS functionals and their TS corrections, respectively. Without the TS contribution [Fig. 8(b)], all functionals predict a basically unbound pentane dimer due to the lack of the long-range vdW part in the PBE hybrid family of XC functionals. Near the equilibrium geometry, all three functionals give binding curves that differ only slightly and, hence, are not the main reason for the trend observed in Fig. 8(a). As a consequence, the difference in the binding curves of Fig. 8(a) must arise predominantly from the TS correction.

Indeed, Fig. 8(b) shows that the TS correction differs significantly between the individual functionals, in particular in a regime near the equilibrium distance. This $\alpha$ dependence originates either from the density dependence of the $C_{6}[\rho]$ coefficients [Eq. (36)] or from the range separation parameter $s_{R}$ in the damping function [Eq. (37)]. Figure 8(d) shows the TS correction for all three functionals where the $s_{R}$ parameter is set to one. All three curves are essentially on top of each 
TABLE II. Range seperation parameter $s_{R}$ and MAPEs for the S22 test set [103] for the $\operatorname{PBE}, \operatorname{PBE0}$, and $\operatorname{PBEh}\left(\alpha^{*}\right)$ functionals. The errors are relative to $\operatorname{CCSD}(\mathrm{T})$ extrapolated to the complete basis set limit taken from Ref. [104].

\begin{tabular}{lccrrr}
\hline \hline & $s_{R}$ & H bonds & Dispersion & Mixed & Total \\
\hline PBE & 0.94 & $8.1 \%$ & $106.7 \%$ & $51.8 \%$ & $57.8 \%$ \\
PBE0 & 0.96 & $7.3 \%$ & $104.6 \%$ & $46.8 \%$ & $55.2 \%$ \\
PBEh $\left(\alpha^{*}\right)$ & 1.02 & $6.4 \%$ & $96.6 \%$ & $32.2 \%$ & $47.8 \%$ \\
\hline \hline
\end{tabular}

other, implying that the $C_{6}$ coefficients do not change with $\alpha$. Thus, it is the $s_{R}$ parameter in the damping function that causes the difference in the curves of Figs. 8(c) and, hence, of Fig. 8(a).

The $s_{R}$ parameter is the empirical part of the TS method. In the TS scheme [30], the $s_{R}$ parameter is obtained by a fit to $\operatorname{CCSD}(\mathrm{T})$ reference data extrapolated to the complete basis set limit [104] of the S22 data set [103]. The $s_{R}$ fit needs to be performed for each functional; the ensuing $s_{R}$ values are collected in Table II.

The $s_{R}$ parameter increases as a function of $\alpha$ from 0.94 (PBE) to $1.02\left[\operatorname{PBEh}\left(\alpha^{*}\right)\right]$. Since $s_{R}$ determines the onset of the vdW $\operatorname{PBEh}\left(\alpha^{*}\right)+\mathrm{TS}$ becomes less attractive and alleviates the overbinding behavior of PBE+TS or PBE0+TS.

The reason for an increase of $s_{R}$ upon increasing $\alpha$ is also given in Table II. In spite of the large error in describing interaction energies of the PBE hybrid family of $\mathrm{XC}$ functionals when dispersion contributions are important, there is a clear trend: the error reduces for all bonding types present in the S22 upon increasing $\alpha$. Now, in the TS scheme, $s_{R}$ is obtained by fitting to the S22 test set. If the underlying functional is already in better agreement with the $\operatorname{CCSD}(\mathrm{T})$ reference data, the vdW contribution reduces; within the TS formalism, this can only be achieved by "pushing" the vdW tail further out via increasing the $s_{R}$ parameter in the damping function. Hence, $\operatorname{PBEh}(\alpha)$ has the largest $s_{R}$ parameter among all considered functionals.

\section{2. $r P T 2$}

The benzene dimer, item number 24 of the S66 data set, was identified by Ren et al. [31,32] as a challenging system for RPA based methods. We therefore adopt it here to analyze the starting point dependence of rPT2. Figure 9 shows the computed binding curves, whereas the reference $\operatorname{CCSD}(\mathrm{T})$ curve was taken from the $S 66 \times 8$ data set [26].

Near the equilibrium distance, rPT2@PBE [Fig. 9(a)] underestimates the binding energy by about $30 \%$. An increase of $\alpha$ in the DFT functional amplifies the underbinding behavior of $\mathrm{rPT} 2\left(\alpha^{*}=0.77\right.$ for the benzene dimer). To gain more insight, we split the rPT2 total energy into its contributions according to Eqs. (38) and (39). Figures 9(c) and 9(d) display the corresponding SOSEX and rSE corrections. While all starting points give the same repulsive SOSEX contribution, rSE acts slightly attractive for PBE but becomes repulsive for the hybrid starting points. For PBE reference states, SOSEX and rSE tend to cancel each other, while they add up in a repulsive fashion otherwise. As a consequence, rPT2 does not
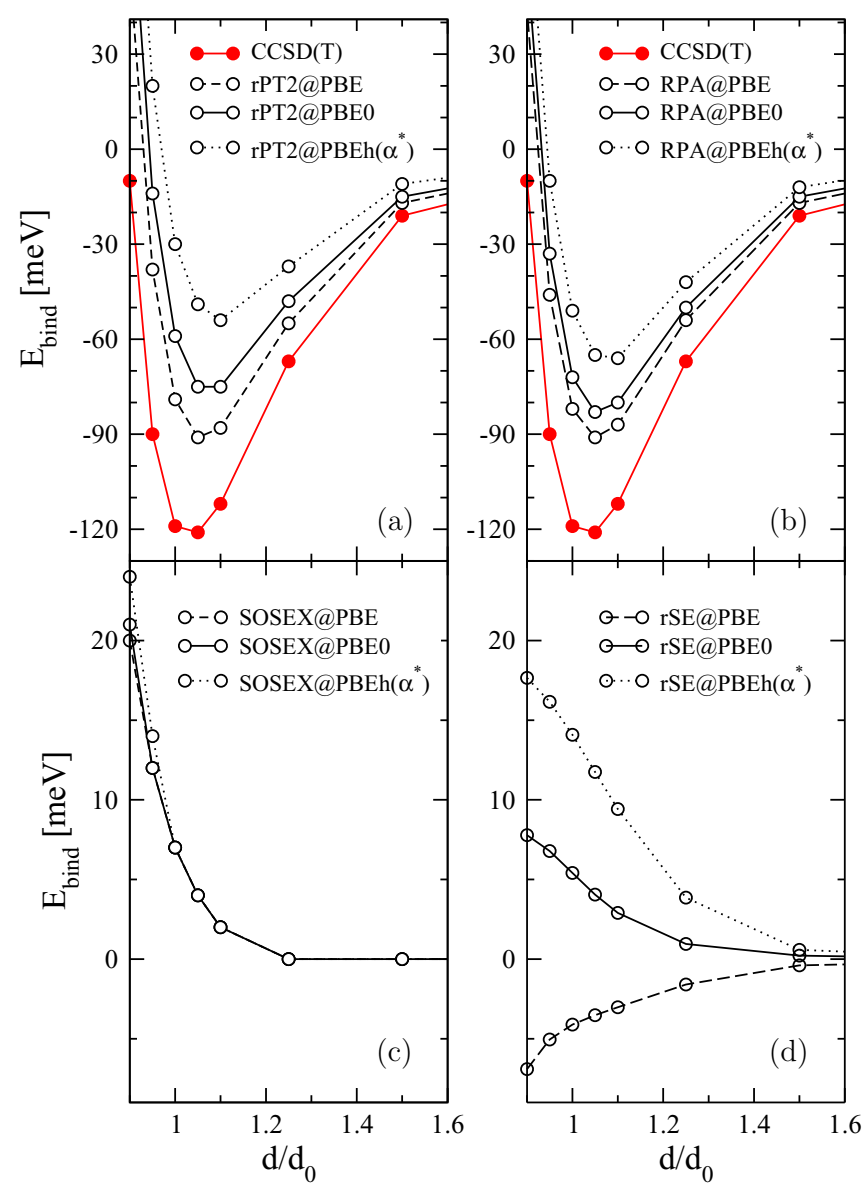

FIG. 9. Binding curve for the benzene dimer obtained by (a) rPT2, (b) EX+cRPA. (c) Shows the SOSEX and (d) the rSE correction. Also shown is the $\operatorname{CCSD}(\mathrm{T})$ reference curve, which was taken from Ref. [26]. The binding distance is given by the scaling parameter $d / d_{0}$ with respect to the equilibrium distance.

improve over EX+cRPA for PBE input orbitals and performs worse for hybrid orbitals.

Also, EX+cRPA exhibits a performance loss upon increasing $\alpha$ as shown in Fig. 9(b). The $\alpha$ dependence of the exchange-only (EX) contribution and the RPA correlation energy (cRPA) to the binding energy is shown in Fig. 10(a).

Plotted are the differences with respect to the PBE starting point

$$
\begin{array}{r}
\triangle \mathrm{EX} @ \mathrm{PBE} 0:=\mathrm{EX} @ \mathrm{PBE} 0-\mathrm{EX} @ \mathrm{PBE}, \\
\triangle \mathrm{cRPA} @ \mathrm{PBE} 0:=\mathrm{cRPA} @ \mathrm{PBE} 0-\mathrm{cRPA} @ \mathrm{PBE},
\end{array}
$$

and similar for the $\mathrm{PBE} 0$ and $\operatorname{PBEh}\left(\alpha^{*}\right)$ functionals. Increasing $\alpha$ renders cRPA less attractive, while at the same time the EX contribution gets less repulsive. These $\alpha$-induced deviations from EX@PBE and cRPA@PBE cancel each other only partly; in particular, the cRPA trend towards weaker binding energies dominates the opposite trend of EX. Thus, $\mathrm{EX}+\mathrm{cRPA}$ decreases the intermolecular interaction strength as $\alpha$ increases.

Returning to the rSE starting point dependence, Fig. 10(a) also shows $\triangle \mathrm{EX} @ \mathrm{HF}=\mathrm{EX} @ \mathrm{HF}-\mathrm{EX} @ \mathrm{PBE} . \mathrm{As}$ 

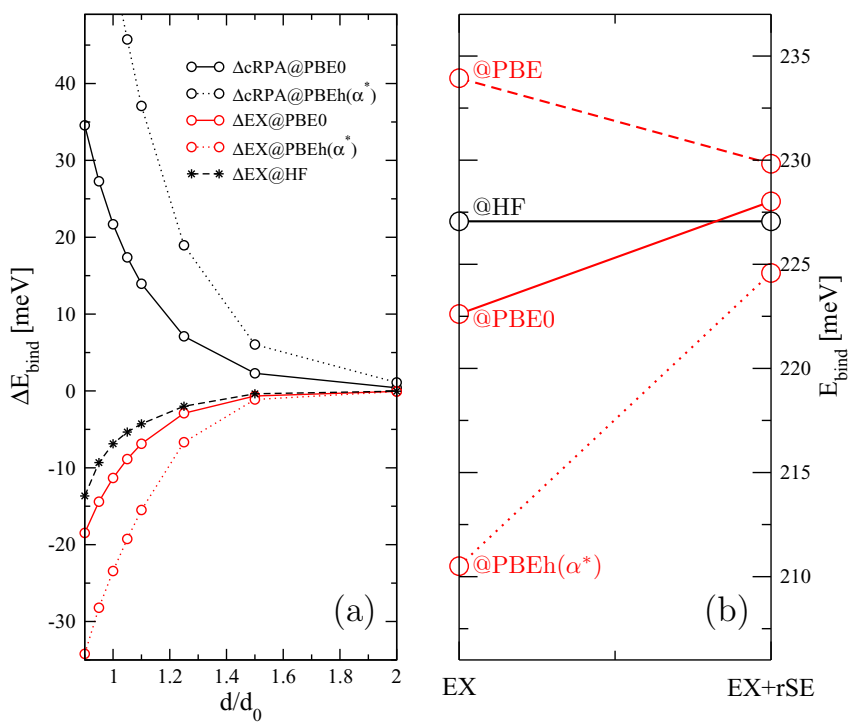

FIG. 10. EX and RPA contributions for the benzene-benzene binding energy (a). Shown are the differences with respect to the PBE starting point [Eqs. (41) and (42)]. (b) Shows the rSE contributions to EX at the equilibrium distance $d / d_{0}=1$.

previously observed by Ren et al. [109], EX@HF is more attractive than EX@PBE. The rSE correction developed by Ren et al. [32,109] encompasses this difference between EX@HF and EX@PBE. For HF, rSE is zero (Brillouin's theorem $[109,110])$. rSE thus corrects towards the HF total energy. This is shown in Fig. 10(b) for the equlibrium distance $d / d_{0}=1$ of the benzene dimer. However, for PBE0 and $\operatorname{PBEh}\left(\alpha^{*}\right)$ starting points, the EX contribution to the binding energy is already lower than in HF. As a consequence, correcting towards HF via rSE acts repulsive because it shifts the binding energy towards larger values. Thus, the inclusion of rSE is only beneficial for the PBE starting point. It does not improve the notorious underbinding behavior of RPA for functionals for which the EX contribution to the binding energy is already lower than HF.

\section{B. Atomization energies}

We now investigate atomization energies for the G2-1 test set [27]. The set contains 55 small molecules with experimental reference values that are corrected for zero-point effects [111].

Figure 11(a) shows the MAPEs for the PBE $(\alpha=0)$, $\operatorname{PBEO}\left(\alpha=\frac{1}{4}\right)$, and $\operatorname{PBEh}(\alpha *)$ functionals within the GKS scheme. Also included are the rPT2 results for the PBE, PBE0,

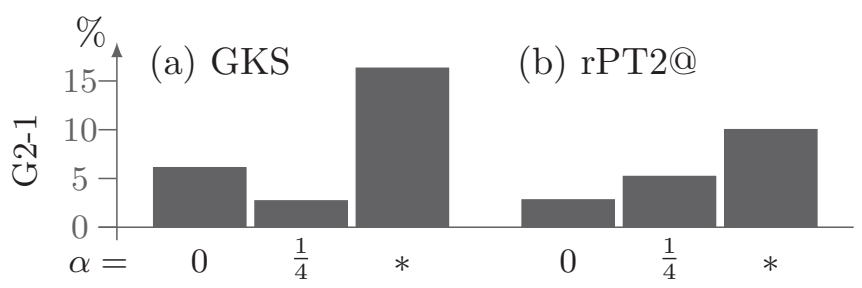

FIG. 11. MAPE (\%) for the G2 test set of atomization energies [27] for GKS, rPT2. Reference data are from Ref. [111]. The results were obtained from the Gaussian cc-pV6Z basis set [95]. and $\operatorname{PBEh}\left(\alpha^{*}\right)$ starting points. Following Ren et al. [31,32], a Gaussian cc-pV6Z basis set [95] was used and a counterpoise correction performed [105]. EX+cRPA@PBE and r2PT@PBE results for the G2-1 have been published by Paier et al. [112,113] and Ren et al. [31,32].

rPT2 strongly depends on the underlying XC functional. Among the three considered starting points, rPT2@PBE shows the best performance with a MAPE of $2.8 \%$. Increasing the fraction of exact exchange $\alpha$ in the DFT functional results in an underestimation of the atomization energies. The pronounced $\mathrm{rPT} 2$ starting point dependence emerges from both the rSE and SOSEX corrections to EX+cRPA. EX+cRPA itself depends relatively weakly on the starting point but it has an overall tendency to underestimate atomization energies. That EX+cRPA@PBE underestimates atomization energies has been first observed by Furche [114] and repeatedly reported in the literature for the G2-1 set $[31,32,112,113]$. The inclusion of the rSE correction increases atomization energies and thus appears to be beneficial, irrespective of the underlying functional. However, the magnitude of the rSE correction depends sensitively on the functional and reduces upon increasing $\alpha$. For the $\operatorname{PBEh}\left(\alpha^{*}\right)$ functional, in particular, the rSE correction is very small and only leads to a very small improvement of $0.2 \%$ compared to EX+cRPA@ $\operatorname{PBEh}\left(\alpha^{*}\right)$.

Unlike in S66, the SOSEX correction depends more strongly on the starting point. SOSEX@PBE0 or $\operatorname{SOSEX} @ \operatorname{PBEh}\left(\alpha^{*}\right)$ tend to decrease atomization energies. As a consequence, adding SOSEX to EX+cRPA degrades the description of atomization energies for these two starting points. While this effect is negligible for SOSEX@PBE0 (the MAPE increases by $0.2 \%$ compared to EX+cRPA+rSE@PBE0), it amounts to a $2.3 \%$ higher $\operatorname{MAPE}$ for the $\operatorname{PBEh}\left(\alpha^{*}\right)$ starting point [compared to $\left.\mathrm{EX}+\mathrm{cRPA}+\mathrm{rSE} @ \operatorname{PBEh}\left(\alpha^{*}\right)\right]$. On the other hand, SOSEX@PBE increases atomization energies, with a reduction in error that is similar than rSE@PBE. As a consequence, adding both (i.e., rPT2@PBE) abates the notorious underestimation of atomization energies in EX+cRPA@PBE. This improvement is reduced for rPT2@PBE0 which still benefits to some extent from the rSE correction, while the SOSEX correction makes rPT2 perform worse than $\mathrm{EX}+\mathrm{cRPA}$ for the $\operatorname{PBEh}\left(\alpha^{*}\right)$ starting point. Thus, among all RPA based methods considered in this study, rPT2@PBE is the most accurate for predicting atomization energies.

At the same time, PBE0 gives the overall most accurate atomization energies for the methods we have considered. PBE0 has a MAPE of $2.7 \%$, which is slightly lower than the 2.8\% MAPE of rPT2@PBE. Figure 11 shows that the error for the PBE hybrid family of XC functionals itself strongly depends on the fraction of exact exchange $\alpha$. PBE tends to overestimate atomization energies, whereas an increase of $\alpha$ decreases atomization energies. For this reason, $\operatorname{PBEh}\left(\alpha^{*}\right)$ underestimates atomization energies. The best performing $\alpha$ value should thus lie in-between zero and $\alpha^{*}$, which is met by $\operatorname{PBE0}\left(\alpha=\frac{1}{4}\right)$.

\section{Reaction barrier heights}

Chemical reaction barrier heights, defined as the energy difference between the reactants and the transition state, play 




FIG. 12. MAPE (\%) of the HTBH38 and NHTBH38 test sets for barrier heights. The errors are relative to reference data of Refs. [28,29] for HTBH38 and NHTBH38, respectively. The results were obtained with the Gaussian cc-pV6Z basis set [95].

a decisive role in the description of chemical reactions. In this work, we consider the HTBH38 [28] and NHTBH38 [29] test sets of Truhlar and co-workers. Each set contains forward and backward barrier heights for 19 hydrogen transfer reactions (HTBH38) and non-hydrogen-transfer reactions (NHTBH38).

Figure 12(a) shows the MAPEs for the PBE $(\alpha=0)$, $\operatorname{PBE0}\left(\alpha=\frac{1}{4}\right)$, and $\operatorname{PBEh}\left(\alpha^{*}\right)$ functionals within the GKS scheme. Figure 12(b) shows the rPT2 results for the PBE, $\operatorname{PBE0}$, and $\operatorname{PBEh}\left(\alpha^{*}\right)$ starting points. The reference data were obtained either using the Weizmann-1 theory [115] or by other "best theoretical estimates," as detailed in Refs. [28,29]. EX+cRPA@PBE has been benchmarked before by Eshuis et al. [116], Paier et al. [113], and Ren et al. [31,32], who also showed rPT2@PBE results. Following the work of Ren et al., we obtained the results with the Gaussian cc-pV6Z basis set [95].

In general, the scale of Fig. 12 implies that all considered methods perform much worse for barrier heights as compared to atomization energies or weak interactions. In particular, non-hydrogen-transfer barrier heights appear problematic. While rPT2@PBE exhibits a slightly better performance than rPT2@PBE0 for HTBH38, the PBE0 starting point appears much more accurate than PBE for NHBH38. Therefore, in contrast to the previous test sets, rPT2@PBE0 shows the best rPT2 performance for barrier heights. However, for all three starting points, adding SOSEX and rSE corrections worsens the results compared to EX+cRPA. On average, adding the rSE correction to EX+cRPA underestimates barrier heights while the SOSEX correction exhibits the opposite behavior. These errors cancel each other partly in rPT2. On the EX+cRPA level, the starting point dependence is most pronounced for non-hydrogen-transfer reactions, for which EX+cRPA@PBE tends to underestimate barrier heights. Increasing $\alpha$ in the PBE hybrid functional increases EX+cRPA barrier heights, $\alpha=\frac{1}{4}$, then gives the best agreement with the reference barrier heights. Further increasing $\alpha$ to $\operatorname{PBEh}(\alpha)$ overshoots quite significantly for non-hydrogen-transfer barrier heights.

The trend of increased barrier heights upon increasing $\alpha$ is already present for the PBE hybrid family itself. PBE tends to underestimate barrier heights, whereas $\operatorname{PBEh}\left(\alpha^{*}\right)$ overestimates them. As a consequence, a functional with an intermediate value of $\alpha$ should give the best compromise.
For the considered set of functionals this is achieved by PBE0, which exhibits the lowest MAPE while still having the tendency to underestimate barrier heights.

\section{ERROR ANALYSIS}

For ionization potentials [12] and weak interaction energies, the performance of $\operatorname{PBEh}\left(\alpha^{*}\right)$ combined with vdW-TS is encouraging. However, this study shows that $\operatorname{PBEh}\left(\alpha^{*}\right)$ degrades the description of atomization energies and barrier heights. Focusing on atomization energies we proceed with an error analysis.

One source of error is the size-consistency error. Size consistency is a sum rule, which states that the total energy of a system that splits into two well-separated subsystems should be equal to the sum of the individual subsystems [117]. A functional that violates this sum rule suffers from the sizeconsistency error. Per construction, the $\operatorname{PBEh}\left(\alpha^{*}\right)$ functional is not size consistent because it uses different $\alpha^{*}$ values for the system and its subsystems. This has been already discussed for optimally tuned range separated hybrid functionals and it has been shown that the size-consistency errors translate into errors in the prediction of atomization energies [118]. In order to estimate this error for the $\operatorname{PBEh}\left(\alpha^{*}\right)$ functional, we compare the errors for the $\mathrm{G} 2$ atomization energies [27] in two different computational setups (Fig. 13). In one setup, we calculate the atomization energies with the original $\operatorname{PBEh}\left(\alpha^{*}\right)$ functional, i.e., we use for the molecule and its atoms their individual $\alpha_{\text {mol }}^{*}$ and $\alpha_{\text {atom }}^{*}$ values, respectively [ $*$ in Fig. 13(b)]. In the other setup, we calculate the atomization energies using the $\alpha_{\mathrm{mol}}^{*}$ from the molecule also for the atoms [ $\alpha_{\mathrm{mol}}^{*}$ in Fig. 13(a)]. It can be seen that the error reduces from $16.3 \%$ to $12 \%$, implying that this error is sizable also for the PBE hybrid family.

However, the residual error is still much larger than for PBE or PBE0. To analyze the discrepancy between PBE0 and $\operatorname{PBEh}\left(\alpha^{*}\right)$, we follow Kim et al. [119,120], and split the error into a density driven and a functional driven contribution. $\operatorname{PBE} 0$ evaluated for the $\operatorname{PBEh}\left(\alpha^{*}\right)$ density $\left[\operatorname{PBE0} @ \operatorname{PBEh}\left(\alpha^{*}\right)\right.$, Fig. 13(b)] gives virtually the same performance as PBE0. This implies that the $\operatorname{PBEh}\left(\alpha^{*}\right)$ density is not responsible for the performance loss of $\operatorname{PBEh}\left(\alpha^{*}\right)$. Thus, it is the functional form of $\operatorname{PBEh}\left(\alpha^{*}\right)$ that causes the poor description of atomization

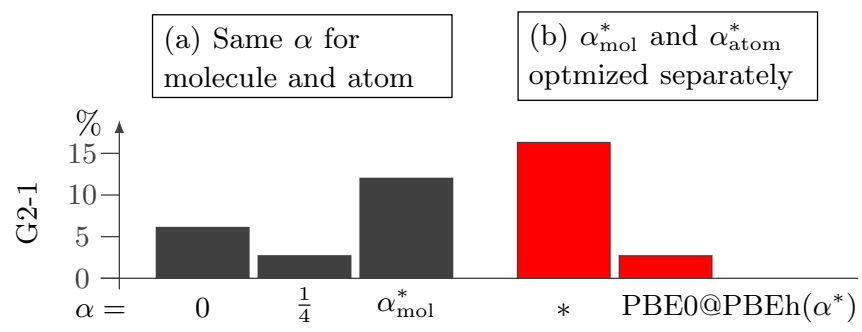

FIG. 13. MAPE (\%) for the G2 test set of atomization energies [27] for (a) functionals that use the same $\alpha$ value for molecules and atoms: $\operatorname{PBE}(\alpha=0), \operatorname{PBE} 0\left(\alpha=\frac{1}{4}\right)$, and $\operatorname{PBEh}\left(\alpha_{\text {mol }}^{*}\right)$ using the optimum $\alpha$ value of the molecule $\left(\alpha_{\text {mol }}^{*}\right)$. (b) Shows the MAPE where $\alpha$ was individually optimized for molecules and atoms $\left(\alpha_{\text {atom }}^{*}\right)$. $\operatorname{PBE0} @ \operatorname{PBEh}\left(\alpha^{*}\right)$ is the PBE0 energy expression evaluated for $\operatorname{PBEh}\left(\alpha^{*}\right)$ densities. Reference data are from Ref. [111]. 
energies. The reason is probably that the PBE hybrid family is too restricted. In particular, it neglects the $\alpha$ dependence of the correlation energy, which should be present in the exact GKS formalism [121]. Thus, constructing functionals that are compatible with a high fraction of exact exchange that yields both good binding energetics and physical meaningful frontier eigenvalues may be regarded as a challenge for future functional development [122,123].

\section{SUMMARY}

In summary, we have investigated the deviation from straight line error for the PBE hybrid family of XC functionals. We found in agreement with previous studies [19] that relatively high $\alpha$ values of $0.7 \sim 0.8$ of fraction of exact exchange are needed to restore the straight line condition for the TTF and TCNQ molecules. We have further demonstrated that the deviation from straight line error can lead to severe artifacts in the description of electron transfer and interaction energies for the TTF-TCNQ complex. These can be cured by using higher $\alpha$ values. In particular, we have found that our recently proposed $\operatorname{PBEh}\left(\alpha^{*}\right)$ method (that obtains $\alpha^{*}$ via the $G W$ self-energy) significantly reduces the deviation from straight line error compared to PBE and PBE0, but has a residual concavity error. We have assessed the performance and the compatibility with the Tkatchenko-Scheffler scheme and rPT2 method of PBE, PBE0, and $\operatorname{PBEh}\left(\alpha^{*}\right)$. We found that the Tkatchenko-Scheffler scheme is compatible with $\operatorname{PBEh}\left(\alpha^{*}\right)$ for weakly interacting systems, whereas rPT2 exhibits a significant performance loss for the $\operatorname{PBEh}\left(\alpha^{*}\right)$ starting point. Furthermore, $\operatorname{PBEh}\left(\alpha^{*}\right)$ is not adequate in describing barrier heights and atomization energies and is clearly outperformed by PBE0.

\section{ACKNOWLEDGMENTS}

We thank A. Tkatchenko for calculating the $s_{R}$ parameter for $\operatorname{PBEh}\left(\alpha^{*}\right)$. V.A. acknowledges interesting discussions with G. Heimel. P.R. acknowledges financial support from the Academy of Finland through its Centers of Excellence Program (Projects No. 251748 and No. 284621).
[1] C. Adamo and V. Barone, J. Chem. Phys. 110, 6158 (1999).

[2] J. Heyd, G. E. Scuseria, and M. Ernzerhof, J. Chem. Phys. 118, 8207 (2003).

[3] J. Heyd, G. E. Scuseria, and M. Ernzerhof, J. Chem. Phys. 124, 219906 (2006)

[4] J. Paier, M. Marsman, K. Hummer, G. Kresse, I. C. Gerber, and J. G. Ngyn, J. Chem. Phys. 124, 154709 (2006).

[5] J. E. Moussa, P. A. Schultz, and J. R. Chelikowsky, J. Chem. Phys. 136, 204117 (2012).

[6] J. P. Perdew, K. Burke, and M. Ernzerhof, Phys. Rev. Lett. 77, 3865 (1996).

[7] J. P. Perdew, M. Ernzerhof, and K. Burke, J. Chem. Phys. 105, 9982 (1996).

[8] A. Alkauskas, P. Broqvist, and A. Pasquarello, Phys. Status Solidi B 248, 775 (2011).

[9] Y. Zhao and D. G. Truhlar, Acc. Chem. Res. 41, 157 (2008).

[10] A. D. Becke, J. Chem. Phys. 98, 1372 (1993).

[11] L. Hedin, Phys. Rev. 139, A796 (1965).

[12] V. Atalla, M. Yoon, F. Caruso, P. Rinke, and M. Scheffler, Phys. Rev. B 88, 165122 (2013).

[13] F. Caruso, V. Atalla, X. Ren, A. Rubio, M. Scheffler, and P. Rinke, Phys. Rev. B 90, 085141 (2014).

[14] M. Pinheiro, M. J. Caldas, P. Rinke, V. Blum, and M. Scheffler, Phys. Rev. B 92, 195134 (2015).

[15] N. A. Richter, S. Sicolo, S. V. Levchenko, J. Sauer, and M. Scheffler, Phys. Rev. Lett. 111, 045502 (2013).

[16] A. Seidl, A. Görling, P. Vogl, J. A. Majewski, and M. Levy, Phys. Rev. B 53, 3764 (1996).

[17] J. P. Perdew, R. G. Parr, M. Levy, and J. L. Balduz, Phys. Rev. Lett. 49, 1691 (1982).

[18] W. Yang, A. J. Cohen, and P. Mori-Sánchez, J. Chem. Phys. 136, 204111 (2012).

[19] N. Sai, P. F. Barbara, and K. Leung, Phys. Rev. Lett. 106, 226403 (2011)
[20] J. D. Gledhill, M. J. G. Peach, and D. J. Tozer, J. Chem. Theory Comput. 9, 4414 (2013).

[21] T. Stein, H. Eisenberg, L. Kronik, and R. Baer, Phys. Rev. Lett. 105, 266802 (2010).

[22] Y. Imamura, R. Kobayashi, and H. Nakai, Chem. Phys. Lett. 513, 130 (2011).

[23] H. Sun and J. Autschbach, ChemPhysChem 14, 2450 (2013).

[24] R. Baer and D. Neuhauser, Phys. Rev. Lett. 94, 043002 (2005).

[25] E. Livshits and R. Baer, Phys. Chem. Chem. Phys. 9, 2932 (2007).

[26] J. Rezac, K. E. Riley, and P. Hobza, J. Chem. Theory Comput. 7, 2427 (2011).

[27] L. A. Curtiss, K. Raghavachari, P. C. Redfern, and J. A. Pople, J. Chem. Phys. 106, 1063 (1997).

[28] Y. Zhao and D. G. Truhlar, J. Chem. Phys. 125, 194101 (2006).

[29] Y. Zhao, N. Gonzlez-Garca, and D. G. Truhlar, J. Phys. Chem. A 109, 2012 (2005).

[30] A. Tkatchenko and M. Scheffler, Phys. Rev. Lett. 102, 073005 (2009).

[31] X. Ren, P. Rinke, C. Joas, and M. Scheffler, J. Mater. Sci. 47, 7447 (2012).

[32] X. Ren, P. Rinke, G. E. Scuseria, and M. Scheffler, Phys. Rev. B 88, 035120 (2013).

[33] J. F. Janak, Phys. Rev. B 18, 7165 (1978).

[34] M. M. Valiev and G. W. Fernando, Phys. Rev. B 52, 10697 (1995).

[35] T. Koopmans, Physica (Amsterdam) 1, 104 (1934).

[36] C.-O. Almbladh and U. von Barth, Phys. Rev. B 31, 3231 (1985)

[37] J. P. Perdew and M. Levy, Phys. Rev. B 56, 16021 (1997).

[38] M. Levy, J. P. Perdew, and V. Sahni, Phys. Rev. A 30, 2745 (1984).

[39] O. V. Gritsenko and E. J. Baerends, J. Chem. Phys. 120, 8364 (2004). 
[40] O. V. Gritsenko and E. J. Baerends, J. Chem. Phys. 117, 9154 (2002).

[41] D. P. Chong, O. V. Gritsenko, and E. J. Baerends, J. Chem. Phys. 116, 1760 (2002).

[42] O. V. Gritsenko, B. Brada, and E. J. Baerends, J. Chem. Phys. 119, 1937 (2003).

[43] J. P. Perdew and M. Levy, Phys. Rev. Lett. 51, 1884 (1983).

[44] L. J. Sham and M. Schlüter, Phys. Rev. Lett. 51, 1888 (1983).

[45] W. Kohn, Phys. Rev. B 33, 4331 (1986).

[46] T. Gould and J. Toulouse, Phys. Rev. A 90, 050502 (2014).

[47] P. Mori-Sánchez, A. J. Cohen, and W. Yang, Phys. Rev. Lett. 100, 146401 (2008).

[48] A. J. Cohen, P. Mori-Sánchez, and W. Yang, Science 321, 792 (2008).

[49] A. J. Cohen, P. Mori-Sánchez, and W. Yang, Chem. Rev. 112, 289 (2012).

[50] Y. Zhang and W. Yang, J. Chem. Phys. 109, 2604 (1998).

[51] P. Mori-Sánchez, A. J. Cohen, and W. Yang, J. Chem. Phys. 125, 201102 (2006).

[52] R. Haunschild, T. M. Henderson, C. A. Jimnez-Hoyos, and G. E. Scuseria, J. Chem. Phys. 133, 134116 (2010).

[53] O. A. Vydrov, G. E. Scuseria, and J. P. Perdew, J. Chem. Phys. 126, 154109 (2007).

[54] A. Ruzsinszky, J. P. Perdew, G. I. Csonka, O. A. Vydrov, and G. E. Scuseria, J. Chem. Phys. 125, 194112 (2006).

[55] A. J. Cohen, P. Mori-Sánchez, and W. Yang, J. Chem. Phys. 126, 191109 (2007).

[56] J. P. Perdew and A. Zunger, Phys. Rev. B 23, 5048 (1981).

[57] J. Chen, J. B. Krieger, Y. Li, and G. J. Iafrate, Phys. Rev. A 54, 3939 (1996).

[58] D. Hofmann, T. Körzdörfer, and S. Kümmel, Phys. Rev. Lett. 108, 146401 (2012).

[59] R. T. Sharp and G. K. Horton, Phys. Rev. 90, 317 (1953).

[60] S. Kümmel and L. Kronik, Rev. Mod. Phys. 80, 3 (2008).

[61] A. J. Cohen, P. Mori-Sánchez, and W. Yang, Phys. Rev. B 77, 115123 (2008).

[62] X. Zheng, A. J. Cohen, P. Mori-Sánchez, X. Hu, and W. Yang, Phys. Rev. Lett. 107, 026403 (2011).

[63] E. Kraisler and L. Kronik, Phys. Rev. Lett. 110, 126403 (2013).

[64] I. Dabo, A. Ferretti, N. Poilvert, Y. Li, N. Marzari, and M. Cococcioni, Phys. Rev. B 82, 115121 (2010).

[65] C. Li, X. Zheng, A. J. Cohen, P. Mori-Sánchez, and W. Yang, Phys. Rev. Lett. 114, 053001 (2015).

[66] A. Görling, Phys. Rev. B 91, 245120 (2015).

[67] E. Kraisler and L. Kronik, J. Chem. Phys. 140, 18A540 (2014).

[68] E. Kraisler and L. Kronik, Phys. Rev. A 91, 032504 (2015).

[69] E. Kraisler, T. Schmidt, S. Kmmel, and L. Kronik, J. Chem. Phys. 143, 104105 (2015).

[70] S. Lany and A. Zunger, Phys. Rev. B 80, 085202 (2009).

[71] S. Lany and A. Zunger, Phys. Rev. B 81, 205209 (2010).

[72] G. Borghi, A. Ferretti, N. L. Nguyen, I. Dabo, and N. Marzari, Phys. Rev. B 90, 075135 (2014).

[73] A. Ferretti, I. Dabo, M. Cococcioni, and N. Marzari, Phys. Rev. B 89, 195134 (2014).

[74] M. Cococcioni and S. de Gironcoli, Phys. Rev. B 71, 035105 (2005).

[75] G. Borghi, C.-H. Park, N. L. Nguyen, A. Ferretti, and N. Marzari, Phys. Rev. B 91, 155112 (2015).

[76] B. Sadigh, P. Erhart, and D. Åberg, Phys. Rev. B 92, 075202 (2015).
[77] J. Toulouse, A. Savin, and H.-J. Flad, Int. J. Quantum Chem. 100, 1047 (2004).

[78] L. Kronik, T. Stein, S. Refaely-Abramson, and R. Baer, J. Chem. Theory Comput. 8, 1515 (2012).

[79] M. Srebro and J. Autschbach, J. Chem. Theory Comput. 8, 245 (2012).

[80] M. Srebro and J. Autschbach, J. Phys. Chem. Lett. 3, 576 (2012).

[81] T. Shimazaki and Y. Asai, Chem. Phys. Lett. 466, 91 (2008).

[82] M. A. L. Marques, J. Vidal, M. J. T. Oliveira, L. Reining, and S. Botti, Phys. Rev. B 83, 035119 (2011).

[83] D. Koller, P. Blaha, and F. Tran, J. Phys.: Condens. Matter 25, 435503 (2013).

[84] S. Refaely-Abramson, S. Sharifzadeh, M. Jain, R. Baer, J. B. Neaton, and L. Kronik, Phys. Rev. B 88, 081204 (2013).

[85] V. Vlček, H. R. Eisenberg, G. Steinle-Neumann, L. Kronik, and R. Baer, J. Chem. Phys. 142, 034107 (2015).

[86] C. Mitra, Phys. Status Solidi B 250, 1449 (2013).

[87] M. van Schilfgaarde, T. Kotani, and S. Faleev, Phys. Rev. Lett. 96, 226402 (2006).

[88] T. Körzdörfer and N. Marom, Phys. Rev. B 86, 041110 (2012).

[89] P. Rinke, A. Qteish, J. Neugebauer, C. Freysoldt, and M. Scheffler, New J. Phys. 7, 126 (2005).

[90] F. Fuchs, J. Furthmüller, F. Bechstedt, M. Shishkin, and G. Kresse, Phys. Rev. B 76, 115109 (2007).

[91] F. Bruneval and M. A. L. Marques, J. Chem. Theory Comput. 9, 324 (2013).

[92] F. Caruso, P. Rinke, X. Ren, M. Scheffler, and A. Rubio, Phys. Rev. B 86, 081102 (2012).

[93] V. Blum, R. Gehrke, F. Hanke, P. Havu, V. Havu, X. Ren, K. Reuter, and M. Scheffler, Comput. Phys. Commun. 180, 2175 (2009).

[94] X. Ren, P. Rinke, V. Blum, J. Wieferink, A. Tkatchenko, A. Sanfilippo, K. Reuter, and M. Scheffler, New J. Phys. 14, 053020 (2012).

[95] T. H. Dunning, J. Chem. Phys. 90, 1007 (1989).

[96] I. Y. Zhang, X. Ren, P. Rinke, V. Blum, and M. Scheffler, New J. Phys. 15, 123033 (2013).

[97] J. C. Slater, Statistical Exchange-Correlation in the SelfConsistent Field (Academic Press, New York, 1972), pp. 1-92.

[98] D. A. Liberman, Phys. Rev. B 62, 6851 (2000).

[99] L. G. Ferreira, M. Marques, and L. K. Teles, AIP Adv. 1, 032119 (2011).

[100] L. Hedin, J. Phys.: Condens. Matter 11, R489 (1999).

[101] F. Hirshfeld, Theor. Chim. Acta 44, 129 (1977).

[102] F. Caruso, P. Rinke, X. Ren, A. Rubio, and M. Scheffler, Phys. Rev. B 88, 075105 (2013).

[103] P. Jurecka, J. Sponer, J. Cerny, and P. Hobza, Phys. Chem. Chem. Phys. 8, 1985 (2006).

[104] T. Takatani et al., J. Chem. Phys. 132, 144104 (2010).

[105] S. Boys and F. Bernardi, Mol. Phys. 19, 553 (2006).

[106] P. Agrawal, A. Tkatchenko, and L. Kronik, J. Chem. Theory Comput. 9, 3473 (2013).

[107] A. M. Reilly and A. Tkatchenko, J. Phys. Chem. Lett. 4, 1028 (2013).

[108] A. Ambrosetti, A. M. Reilly, R. A. DiStasio, and A. Tkatchenko, J. Chem. Phys. 18A508, 140 (2014). 
[109] X. Ren, A. Tkatchenko, P. Rinke, and M. Scheffler, Phys. Rev. Lett. 106, 153003 (2011).

[110] A. Szabo and N. S. Ostlund, Modern Quantum Chemistry: Introduction to Advanced Electronic Structure Theory, Dover Books on Chemistry (Dover, New York, 1996).

[111] D. Feller and K. A. Peterson, J. Chem. Phys. 110, 8384 (1999).

[112] J. Paier, B. G. Janesko, T. M. Henderson, G. E. Scuseria, A. Grneis, and G. Kresse, J. Chem. Phys. 132, 094103 (2010).

[113] J. Paier, X. Ren, P. Rinke, G. E. Scuseria, A. Grneis, G. Kresse, and M. Scheffler, New J. Phys. 14, 043002 (2012).

[114] F. Furche, Phys. Rev. B 64, 195120 (2001).

[115] J. M. L. Martin and G. de Oliveira, J. Chem. Phys. 111, 1843 (1999).
[116] H. Eshuis, J. Bates, and F. Furche, Theor. Chem. Acc. 131, 1 (2012).

[117] J. P. Perdew and S. Kurth, A Primer in Density Functional Theory (Springer, Berlin, 2003), pp. 1-55.

[118] A. Karolewski, L. Kronik, and S. Kümmel, J. Chem. Phys. 138, 204115 (2013).

[119] M.-C. Kim, E. Sim, and K. Burke, Phys. Rev. Lett. 111, 073003 (2013).

[120] M.-C. Kim, E. Sim, and K. Burke, J. Chem. Phys. 140, 18A528 (2014).

[121] A. Grling and M. Levy, J. Chem. Phys. 106, 2675 (1997).

[122] T. Schmidt, E. Kraisler, A. Makmal, L. Kronik, and S. Kümmel, J. Chem. Phys. 140, 18A510 (2014).

[123] T. Schmidt, E. Kraisler, L. Kronik, and S. Kümmel, Phys. Chem. Chem. Phys. 14357, 16 (2014). 